\title{
Investigations of the Origins of Metastable Light-Induced Changes in Hydrogenated Amorphous Silicon
}

\section{Final Subcontract Report}

1 April 1988 - 31 March 1991

\section{J.D. Cohen}

Department of Physics and

Materials Science Institute

University of Oregon

Eugene, Oregon

NREL technical monitor: B. Stafford

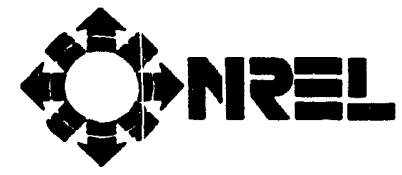

National Renewable Energy Laboratory

(formerly the Solar Energy Research Institute) 1617 Cole Boulevard

Golden, Colorado 80401-3393

A Division of Midwest Research Institute Operated for the U.S. Department of Energy under Contract No. DE-AC02-83CH10093

Prepared under Subcontract No. XM-8-18061-1

December 1991

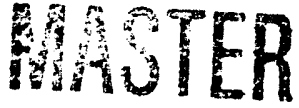


This publication was reproduced from the best available camera-ready copy submitted by the subcontractor and received no editorial review at NREL.

\section{On September 16, 1991, the Solar Energy Research Instltute was designated a national laboratory, and its name was changed to the National Renewable Energy Laboratoy.}

\section{NOTICE}

This report was prepared as an account of work sponsored by an agency of the United States government. Neither the United States government nor any agency thereof, nor any of their employees, makes any warranty, express or implied. or assumes any legal liability or responsibility for the accuracy. com. pleteness, or usefulness of any information. apparatus, product, or process disclosed. or represents that its use would not infringe privately owned rights. Reference herein to any specific commercial product. process, or service by trade name. trademark, manufacturer, or otherwise does not necessarily constitute or imply its endorsement. recommendation. or favoring by the United States government or any agency thereot. The views and opınions of authors expressed herein do not necessarily state or reflect those of the United States government or any agency thereof.

$$
\begin{gathered}
\text { Printed in the United States of America } \\
\text { Available from: } \\
\text { National Technical Informatıon Service } \\
\text { U.S. Department of Commerce } \\
5285 \text { Pon Royal Road } \\
\text { Springfield, VA } 22161 \\
\text { Price: Microfiche A01 } \\
\text { Printed Copy A03 }
\end{gathered}
$$

Codes are used for pricing all publications. The code is determined by the number of pages in the publication. Information pertainıng to the pricing codes can be found in the current issue of the following publications which are generally avallable in most libraries: Energy Research Abstracts (ERA): Govern. ment Reports Announcements and Index (GRA and 1): Scientific and 7echnical Abstract Reports (STAR); and publication NTIS-PR-360 avallable from NTIS at the above address. 
TABLE OF CONTENTS

\section{Page}

LIST OF ILLUSTRATIONS $\ldots \ldots \ldots \ldots \ldots \ldots \ldots \ldots$ ii

LIST OF TABLES $\ldots \ldots \ldots \ldots \ldots \ldots \ldots \ldots \ldots \ldots \ldots \ldots$ iii

EXECUTIVE SUMMARY $\ldots \ldots \ldots \ldots \ldots \ldots \ldots \ldots$ iv

1.0 INTRODUCTION $\ldots \ldots \ldots \ldots \ldots \ldots \ldots \ldots$

2.0 SAMPLES AND SAMPLE TREATMENT

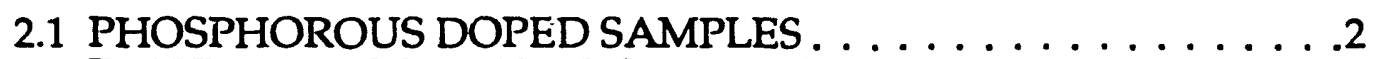

2.2 IMPURITY MODULATED SAMPLES . . . . . . . . .

2.3 PREPARATION OF METASTABLE STATES . . . . . . . . .

3.0 EXPERIMENTAL METHODS

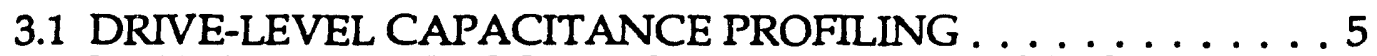

3.2 TRANSIENT PHOTOCAPACITANCE SPECTROSCOPY . . . . . .6

3.3 DEPLETION WIDTH MODULATED ESR SPECTROSCOPY . . . 9

4.0 LIGHT-INDUCED DEFECTS IN SAMPLES WITH MODULATED CARBON IMPURITIES

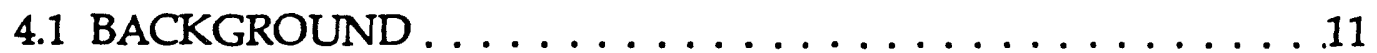

4.2 EXPERIMENTAL RESULTS . . . . . . . . . . . . . . . . . . . . . 11

4.3 NUMERICAL MODELING OF DRIVE-LEVEL RESULTS . . . . 14

4.4 DISCUSSION . . . . . . . . . . . . . . . . 16

5.0 IDENTIFICATION OF DEFECT REACTIONS IN PHOSPHOROUS DOPED SAMPLES

5.1 BACKGROUND . . . . . . . . . . . . . . . . . . . . . . . . . . . .

5.2 EXPERIMENTAL RESULTS . . . . . . . . . . . . . . . . . . . . . . . . . . . . . . . . . . . . .

5.3 DISCUSSION . . . . . . . . . . . . . . . . . . . . 21

6.0 SPIN AND CHARGE STATES ASSOCIATED WITH METASTABLE DEFECTS IN PHOSPHORUS DOPED SAMPLES

6.1 BACKGROUND . . . . . . . . . . . . . . . . . . . . 25

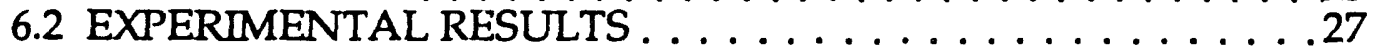

6.3 DISCUSSION . . . . . . . . . . . . . . . . . . . . . . . . 29

7.0 SUMMARY OF KEY FINDINGS AND . . . . . . . . . . . . 31

RECOMMENDATIONS FOR FUTURE STUDY .

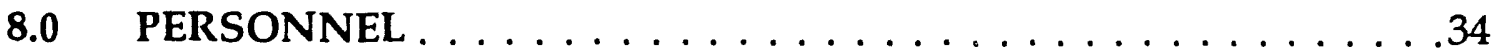

9.0 PUBLISHED PAPERS BASED ON SERI . . . . . . . . . . . . . . . . 34 SUBCONTRACT WORK

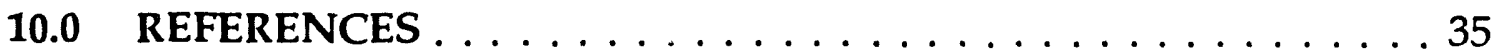




\section{LIST OF ILLUSTRATIONS}

Page

FIG. 1. SIMS deduced profiles for $C, N$, and $H$

in one sample with intentional carbon modulation. . . . . . . . . . . . 4

FIG. 2. The incorporated carbon levels in our a-Si:H samples as a function of the $\mathrm{CH}_{4} / \mathrm{SiH}_{4}$ ratio present in the gas mixture during growth. . . . . . . . . . . .

FIG. 3. Spatial profiles of the occupied bandtail states obtained by the drive-level capacitance profiling method for a $10 \mathrm{Vppm} \mathrm{PH}_{3}$ doped sample in different metastable states . . . . . . . . . . . . . . . .

FIG. 4. Voltage pulse transient photocapacitance spectra taken for an intrinsic a-Si:H sample for

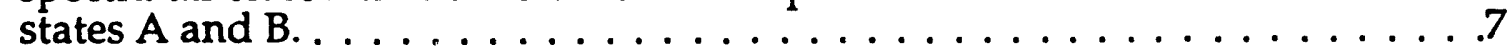

FIG. 5. Measured and calculated drive-level profiles for one sample with a modulated carbon content at two different temperatures for both the dark annealed state A and the light soaked state B of the sample. The SIMS impurity profiles are also displayed along with the deduced spatial dependence of the light-induced metastable defects . . . . . . . . 12

FIG. 6. A series of calculated drive-level profiles for different values of a parameter $\xi$ which determines the abruptness of the change of electronic properties at the boundary between the high and low defect regions. . . . . . . . . . . 15

FIG. 7. Drive-level profiling data for a low carbon modulated sample for states $A$ and $B \ldots \ldots \ldots \ldots$

FIG \&. Total deep defect densities in state A and B deduced for several modulated films as a function of carbon content and for total hydrogen content. . . . . . . . . . . . . . 17

FIG. 9. Change in the deep defect densities $\left(N_{D}\right)$ as a function of the occupied bandtail state density $\left(\mathrm{N}_{\mathrm{BT}}\right)$ for a sequence of metastable states. . . . . . . . . . . . . . 20 
FIG. 10. Voltage pulse transient photocapacitance spectra for a $300 \mathrm{Vppm} \mathrm{PH}_{3}$ doped sample for state $\mathrm{A}$, and also for states following light-soaking and quench cooling . . . . . . . . 22

FIG. 11. Summary of the total deep defect densities vs. phosphorus doping level for state $A$ and state $B$. Also the values of the conduction bandtail occupation for states $\mathrm{A}$ and the quench-cooled states . . . . . . . . . . . . . 22

FIG. 12. DWM-ESR spectra of light-soaked n-type a-Si:H sample showing both the in-phase and quadrature phase signals . . . . . 26

FIG. 13. Spin transient signal of light soaked sample at fixed magnetic field . . . . . . . . . . . . . . . . 26

FIG. 14. Measured and calculated $5 \mathrm{kHz}$ capacitance transients of a partially light-soaked sample, and the deep defect charge signal deduced by calculation $\ldots \ldots \ldots . \ldots 28$

FIG. 15. Comparison of magnitudes of DWM-ESR spin signals and the charge modulation signals for two $\mathrm{PH}_{3}$ doped samples in a variety of metastable states. . . . . . . . . . . 30

\section{LIST OF TABLES}

TABLE I. Comparison of magnitudes and phase angles of spin signals determined from DWM-ESR measurements and charge signals determined from capacitance transient measurements . . . . . . . . . . . . . . . . . . . . . 30 


\section{EXECUTIVE SUMMARY}

During the three years of our Subcontract period, we have continued our investigations of the origins of metastable effects in a-Si:H through three kinds of studies. First of all, the effect of carbon impurities in a-Si:H samples at low concentrations (1at.\% to less than 0.1 at.\%) was investigation using drive-level capacitance profiling (DLCP) measurements on samples whose carbon content was intentionally modulated spatially during growth. We have thus established a strong correlation between the SIMS determined carbon content and the susceptibility of these samples to light-induced metastable defect creation such that a carbon level of 0.5 at. \% leads roughly to an additional 1 to $2 \times 10^{16} \mathrm{~cm}^{-3}$ increase in light-induced defects. No such correlation was found with respect to the variation in total hydrogen content in these samples, nor to their characteristic bandtail (Urbach) energies.

Second, we characterized metastable states in n-type doped a-Si:H samples caused both by quench cooling and by light-soaking with partial annealing. Samples with a $\mathrm{PH}_{3}$ doping between 10 to $300 \mathrm{Vppm}$ were examined. We utilized DLCP measurements to deduce the number of occupied bandtail states $\left(\mathrm{N}_{\mathrm{BT}}\right)$ and transient photocapacitance spectroscopy to determine the density of deep defects $\left(N_{D}\right)$. By correlating the changes in these two quantities with different metastable treatments we have been able to distinguish among many of the basic types of defect reactions that have been proposed. We have found that marked changes in $\mathrm{N}_{\mathrm{BT}}$ are often accompanied by negligible changes in $\mathrm{N}_{\mathrm{D}}$, particularly for quench cooling. Thus we conclude that generally independent mechanisms cause the changes in these two quantities and propose that a dopant activation mechanism, independent of the creation of dangling bonds, must be present.

Finally, we utilized depletion-width-modulated ESR spectroscopy together with junction capacitance transient spectroscopy to investigate deep defect states for various metastable states of a 10 and a $80 \mathrm{Vppm} \mathrm{PH3} \mathrm{doped}$ a-Si:H sample. We were thus able to deduce the changes in total spin and charge associated with the capture and thermal emission of electron from deep mobility gap states. We found that the ratio of the magnitudes of the spin and charge changes in these processes were nearly unity in both samples and for all the metastable states studied, and indicated the predominance of the $D-/ D^{\circ}$ transition from the $D$ center in these samples. However, we also found evidence for a large phase lag of the spin signal relative to the alternating applied bias and also relative to the deduced trapped charge signal. These results possible suggest the existence of an intermediate state involved in the capture or reemission of deep trapped charge. 


\subsection{INTRODUCTION}

Our SERI subcontract work over the last three years has been directed at trying to identify some of the fundamental aspects of light-induced mietastable effects in hydrogenated amorphous silicon (a-Si:H). This issue is one the most significant not only with respect to understanding the fundamental electronic properties of this material, but is also a key limitation in attempts to realize the full potencial of a-Si:H in photovoltaic applications. Our own efforts have been directed towards three types of studies that we believe will help distinguish among a multitude of mechanisms that have been proposed over recent years to account for such metastable behavior of a-Si:H.

First of all, we have examined the effects of specific extrinsic impurities on the susceptibility of undoped a-Si:H samples to light-induced degradation. These studies utilize methods which have much greater sensitivity than attempts to resolve such issues in earlier studies. Due to some early evidence suggesting that carbon contamination had a deleterious effect on a-Si:H stability [1,2], we have concentrated our work on this impurity. We thus added methane to our gas mixtures during film growth so that we could get a periodic variation in the carbon concentration profiles within a series of samples. Junction capacitance methods were then used to obtain a spatial profile of the deep defect concentrations within these samples before and after light soaking. We found quite conclusive evidence for an increase in degradation due to carbon impurities down to at least the 0.1 at.\% level. Our result thus contrasts markedly with previous studies which indicated no effects on degradation for atmospheric impurities below the 5 at.\% level. $[3,4]$ We discuss these results in detail in Section 4.

Second, we have investigated the metastable behavior in phosphorus doped samples produced both by light soaking and quench cooling. We developed a method to distinguish between types of proposed metastable defect reactions that involved the activation of dopants together with the creation of new deep defects. Our results, presented in Section 5, indicate that the metastable changes in deep defects and those involved in dopant activation actually appear to occur essentially independent of each other.

Finally, we employed electron spin resonance (ESR) methods in

conjunction with capacitance spectroscopy in phosphorus doped samples to identify the spin state of deep defects for a series of light-soaked and partial 
annealed metastable states. Here we utilized moderately doped samples such that, after light soaking one could move the Fermi level as deep as $0.7 \mathrm{eV}$ below the conduction band mobility edge, $\mathrm{E}_{\mathrm{C}}$. We were thus interested to learn whether the properties of deep states in such light soaked samples appeared more similar to those observed in intrinsic samples than in n-type samples; i.e., whether one would find a higher proportion of singly occupieci defects or evidence for a change in the effective correlation energy between singly and doubly occupied centers. Our studies, detailed in Section 6, did not find any indications of qualitative changes between the annealed or light-soaked samples. However, we did note some anomalies between the spin and charge signals in these samples.

Before discussing the above mentioned studies in detail we will outline our sample preparation methods (Section 2) and also give a brief account of our experimental techniques (Section 3) We will conclude our report in Section 7 with a summary of our key findings and discuss what we consider to be the most important issues for possible follow up investigations.

\subsection{SAMPLES AND SAMPLE TREATMENT}

\subsection{PHOSPHORUS DOPED SAMPLES}

Two series of phosphorus doped samples were grown by the glow discharge method on heavily doped $\mathrm{p}+$ (boron doped) crystalline silicon substrates. Substrate temperatures were in the range $255 \pm 5^{\circ} \mathrm{C}$ and $\mathrm{rf}$ power levels were roughly $250 \mathrm{~mW} / \mathrm{cm}^{2}$ for all films. Gas ratios of $\mathrm{PH}_{3}$ to $\mathrm{SiH}_{4}$ in our initial series of samples were 30,100, and $300 \mathrm{Vppm}$, and in our second series were 4, 10, 19, 55, and $80 \mathrm{Vppm}$. All gas mixtures were further diluted in Ar during growth at levels of 40-50at\%. Films varied in thickness over the range 1.5 to $3 \mu \mathrm{m}$.

These samples were prepared for junction capacitance measurements by the evaporation of a $0.5 \mathrm{~mm}$ diameter Pd Schottky barrier on the free surface. Some of these samples were also utilized for the depletion modulated ESR measurements described in Section 3. In that case larger area Pd Schottky d.ode contacts were evaporated with dimensions $4 \mathrm{~mm} \times 8 \mathrm{~mm}$. In general all such samples consist of two junctions: the top Pd Schottky barrier and the $\mathrm{p}^{+} \mathrm{n}$ junction at the substrate interface. Either junction culd then be selected for study by choosing the polarity of bias applied to the sample. 


\subsection{IMPURITY MODULATED SAMPLES}

For the our studies of the effects of carbon impurities, series of samples were grown with an intentional variation in carbon content produced by switching on and off $\mathrm{CH}_{4}$ within the growth chamber at the 5-20\% level at a rate which gave a $1000-3000 \AA$ periodic composition modulation within the film. Two series of samples were grown with and without the dilution in $\mathrm{Ar}$ at the 50\% level. The switching of gas mixtures was carried out using two SPDT gas switches so that the total chamber pressure and Ar dilution could be kept constant during growth.

At these $\mathrm{CH}_{4}$ levels, the total carbon content modulation was determined by SIMS analysis to vary between 0.2 to $2 \%$. The carbon incorporation fraction for the same $\mathrm{CH}_{4}$ dilution was increased significantly for the films grown with Ar dilution as indicated in Fig. 1. In Fig. 2 we display one representative SIMS profile in which the hydrogen, carbon, and oxygen profiles are given. We observe that the carbon concentrations typically may vary an order of magnitude between the carbon and carbon poor regions while oxygen (and also nitrogen) levels remain fairly constant. In general we also found that the hydrogen concentrations in the films were also modulated slightly by the methane switching as is evident in Fig. 2. However, the overall hydrogen content was found to be modified differently by Ar dilution than carbon. Because of this, possible ambiguities in assigning the cause of increased degradation to either of these two compositional effects could be sorted out.

Semitransparent $\mathrm{Pd}$ metal contacts of area $0.002 \mathrm{~cm}^{2}$ were evaporated on the top surface of each sample for our junction capacitance measurements.

\subsection{PREPARATION OF METASTABLE STATES}

Our measurements were carried out in a dark-annealed state (state A) and in a light-soaked state (state B). State A was obtained by annealing in the dark for 20 minutes at $473 \mathrm{~K}$ and cooling slowly to room temperature. State $B$ was obtained by exposure to filtered tungsten-halogen light at levels of 200$400 \mathrm{~mW} / \mathrm{cm}^{2}$ for 20 to 100 hours (wavelengths shorter than $6000 \AA$ were filtered out so that light exposure would be roughly uniform throughout the samples). 


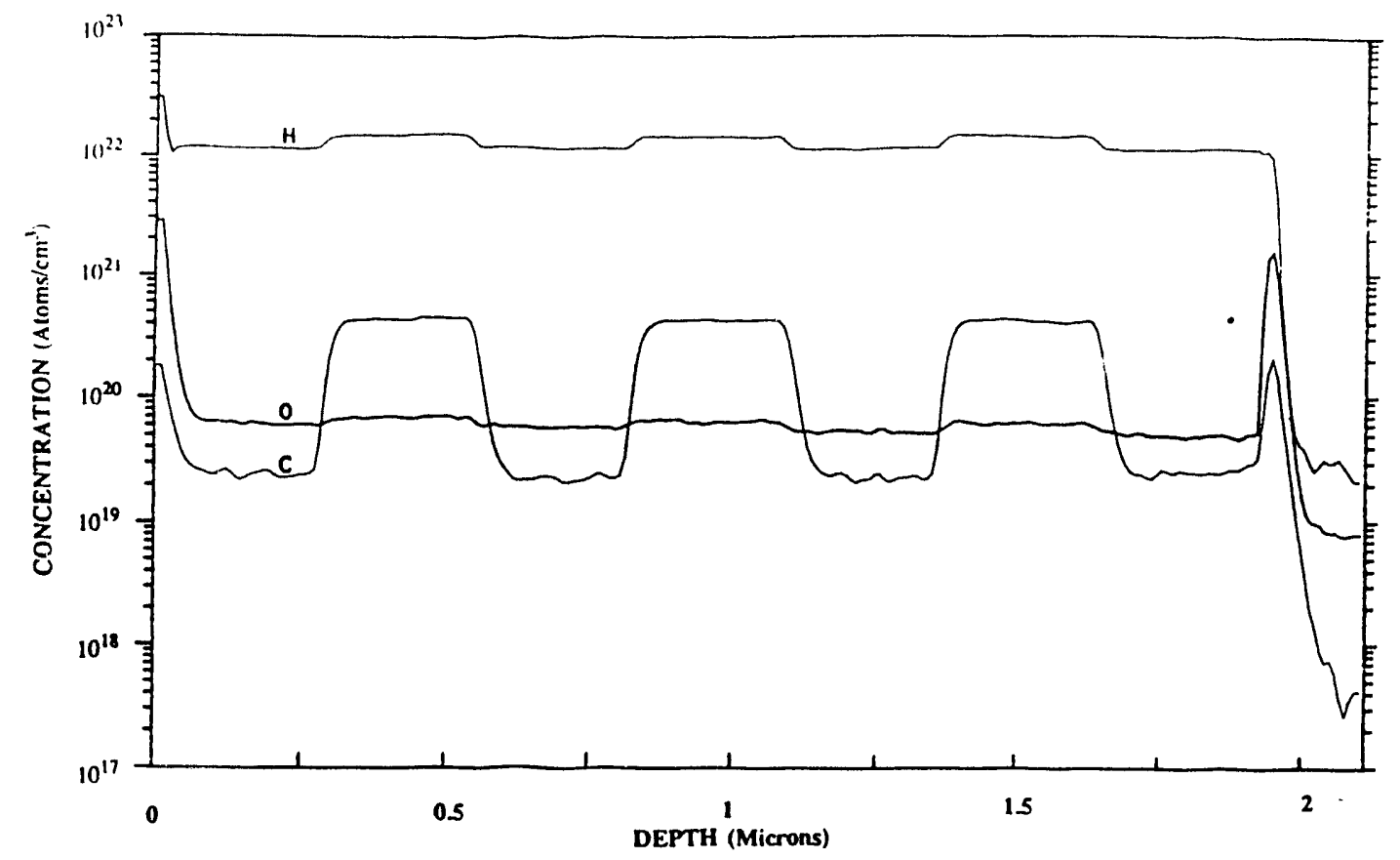

FIG. 1.SIMS deduced profiles for $\mathrm{C}, \mathrm{O}$, and $\mathrm{H}$ in one sample with intentional carbon modulation. The nitrogen contamination level was also profiled but found to be at a much lower level (below $10^{18} \mathrm{~cm}^{-3}$ ). [SIMS profiles courtesy of Sally Asher, SERI]

FIG. 2. The incorporated carbon levels in our a-Si:H samples as a function of the $\mathrm{CH}_{4} / \mathrm{SiH}_{4}$ ratio present in the gas mixture during growth. Note that a higher level of incorporation was obtained when $\mathrm{Ar}$ dilution was employed in the gas mixture.

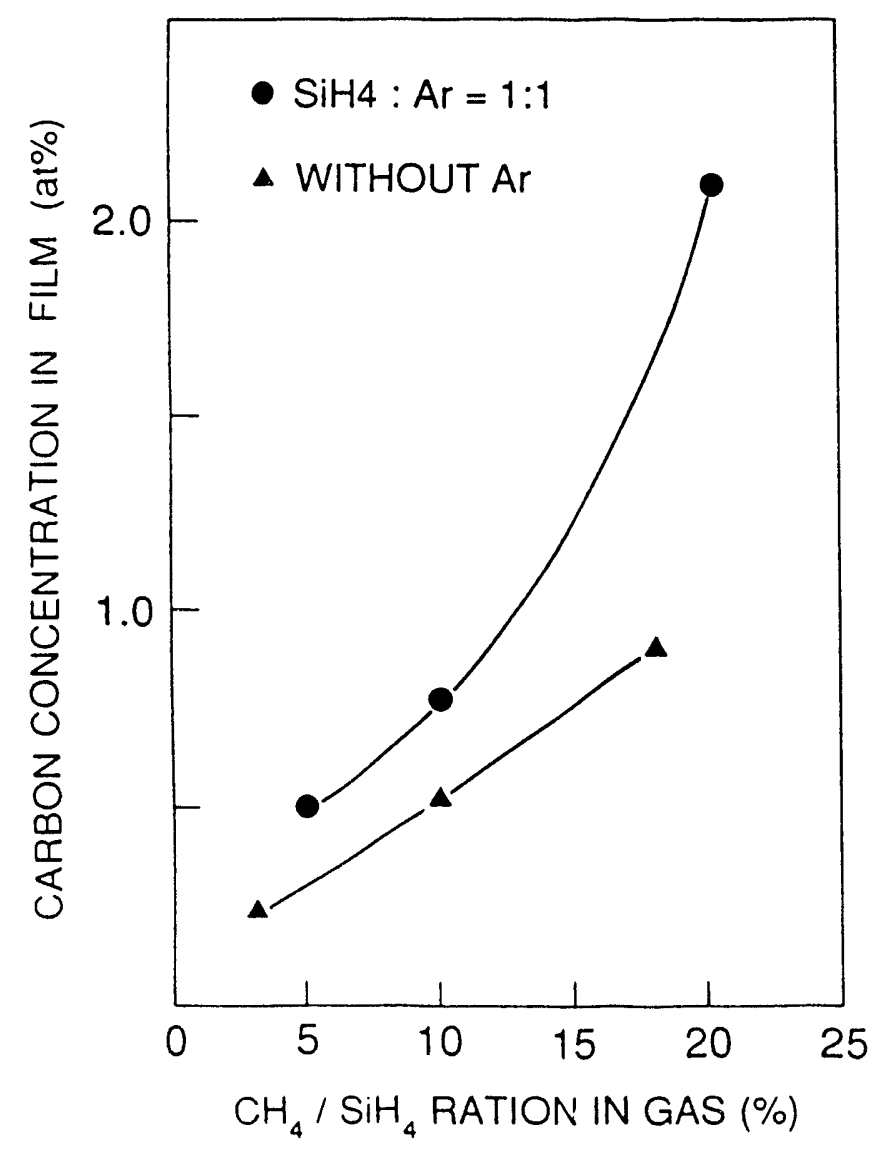


Many of our studies also involved studying samples in several partial anneal "states". These were obtained by heating our light-soaked samples in the dark for 15 minutes at a series of temperatures, typically starting at $350 \mathrm{~K}$ and ending at $470 \mathrm{~K}$ (state $\mathrm{A}$ ) in $30 \mathrm{~K}$ temperature intervals. For our studies of the ntype doped samples other metastable effects, induced by different cooling rates, were also investigated. For these samples "slow" cooling refers to a controlled linear temperature ramp beginning with a 30 minute anneal at $200^{\circ} \mathrm{C}$ and then decreasing the temperature at a rate of $0.1^{\circ} \mathrm{C} / \mathrm{s}$. "Fast" cooled or "quenched" means that samples were cooled from $200^{\circ} \mathrm{C}$ at $7^{\circ} \mathrm{C} / \mathrm{s}$.

\subsection{EXPERIMENTAL METHODS}

\subsection{DRIVE-LEVEL CAPACITANCE PROFILING}

The drive-level capacitance profiling technique was developed in 1984-85 in our laboratory and has been described in several publications $[5,6]$. We will briefly summarize a few of the important features of this method.

Capacitance-voltage (CV) profiling is a standard technique in the characterization of semiconductors which allows one to map out the doping level of a sample versus distance, typically over several tenths of a micron. Unfortunately, due to the high concentration of deep defects over a significant fraction of the gap in a-Si:H, this method fails to be easily interpretable for this material. To overcome this limitation and be able to similarly obtain spatial profiles of gap states in a-Si:H, we developed an alternative technique which examines in detail the dependence of the junction capacitance as a function of applied bias, temperature, plus the peak-to-peak drive amplitude of the alternating component of the junction voltage, $\mathrm{dV}$. In general, one finds that the measured ac capacitance at a particular DC bias depends on $\mathrm{dV}$ according to the relation

$$
C=C_{0}+C_{1} d V+C_{2}(d V)^{2}+\ldots
$$

Thus one experimentally determines the coefficients $C_{0}$ and $C_{1}$. We have shown that a simple algebraic expression using these coefficients then directly yields an integral over the density of gap states [5]:

$$
N_{D L}=\frac{-C_{0}}{2 \varepsilon q A^{2}} C_{1}=\int_{E_{c}-E_{e}}^{E_{F}{ }^{o}} g(E) d E
$$


where $\varepsilon$ is the dielectric constant, $A$ is the junction area, $E_{F}{ }^{O}$ is the Fermi level position in the neutral bulk a-Si:H, $E_{C}$ is the bottom of the conduction band (mobility) edge, and $\mathrm{E}_{\mathrm{e}}$ is the thermal escape depth determined by the temperature, $T$, and (angular) frequency $\omega$ of the capacitance measurement; namely

$$
E_{e}(T, \omega)=k_{B} T \log (v / \omega)
$$

Hence one experimentally obtains an integral over the density of gap states and can spatially profile this energy "slice" of deep defects by varying the $\mathrm{DC}$ applied bias as in conventional $\mathrm{CV}$ profiling and plotting the drive-level density, $\mathrm{N}_{\mathrm{DL}}$ versus $\mathrm{eC}_{0} / \mathrm{A}$. By repeating such profiles at different temperatures one can vary the energy range of the integral and thus obtain $g(E)$ itself.

For our studies of phosphorus doped samples we typically employ a measurement frequency of $1 \mathrm{kHz}$ at a temperature of $240 \mathrm{~K}$. This gives a lower energy cutoff for $E_{e}$ near the bottom of the conduction bandtail at $E_{C}-0.4 \mathrm{eV}$. Thus $\mathrm{N}_{\mathrm{DL}}$ becomes nearly equal to the density of occupied bandtail states, $\mathrm{N}_{\mathrm{BT}}$. Sorne examples of drive-level profiles for different metastable state; of our 10 Vppm sample are given in Fig. 3.

We believe that the drive-level profiling technique represents the most reliable method to separately map out both the spatial and energy variation of the defect density in amorphous silicon films. Thus this method serves as a key part of our studies to correlate the degree of light-induced degradation with selected extrinsic impurities. Also, because the drive-level method gives the gap state occupation as an absolute density, it can serve as a means to calibrate the gap state signals obtained by other measurements, such as those obtained from our photocapacitance spectra, which are not so easily interpreted since they als. depend on optical cross sections for defect transitions.

\subsection{TRANSIENT PHOTOCAPACITANCE SPECTROSCOPY}

We employ photocapacitance spectroscopy to deduce deep defect levels in a-Si:H samples, and thus to examine differences in the energy distributions of these defects for different metastable states of the sample. This method is complementary to the drive-level method in that it allows us to examine $g(E)$ 


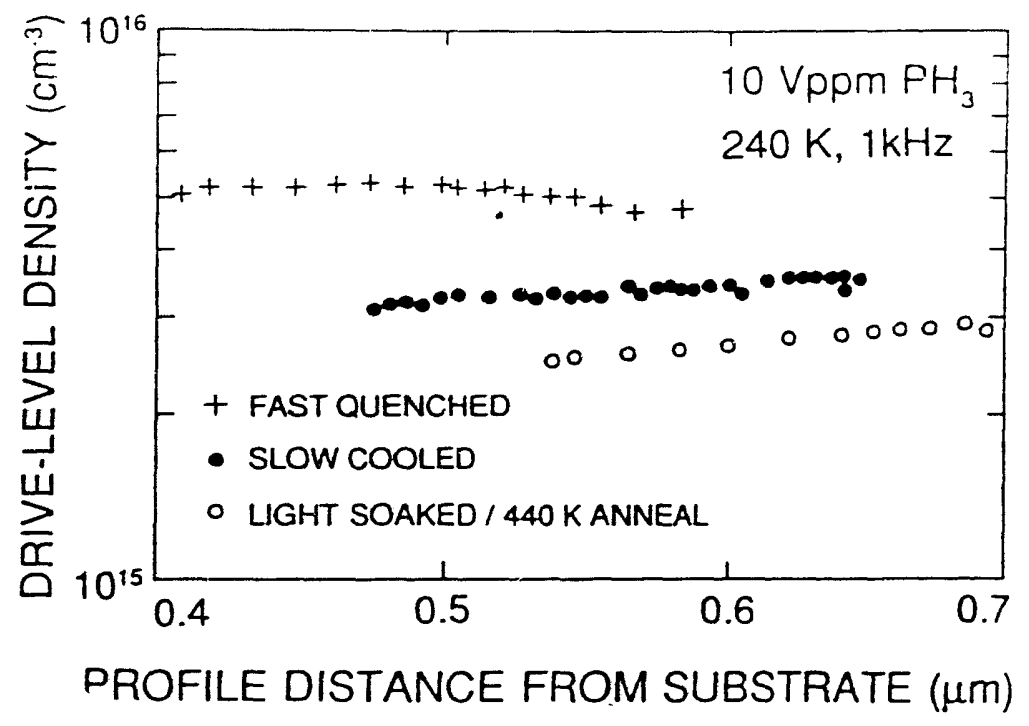

FIG. 3. Spatial profiles of the occupied bandtail states obtained by the drive-level capacitance profiling method for a $10 \mathrm{Vppm} \mathrm{PH}_{3}$ doped sample. Profiles for 3 metastable states are compared: the slow-cooled state (state $\mathrm{A}$ ), the quench cooled state, and a $440 \mathrm{~K}$ partial anneal of the light-soaked state.

FIG. 4. Voltage pulse transient photocapacitance spectra taken for an intrinsic a-Si:H sample for state $A$ and state $B$ at. $380 \mathrm{~K}$ with a frequency of $100 \mathrm{~Hz}$. The total deep defect density for this sample in state A was determined to be $5 \times 10^{15} \mathrm{~cm}^{-3}$ and is thus observed to increase by more than a factor of 3 after light-soaking.

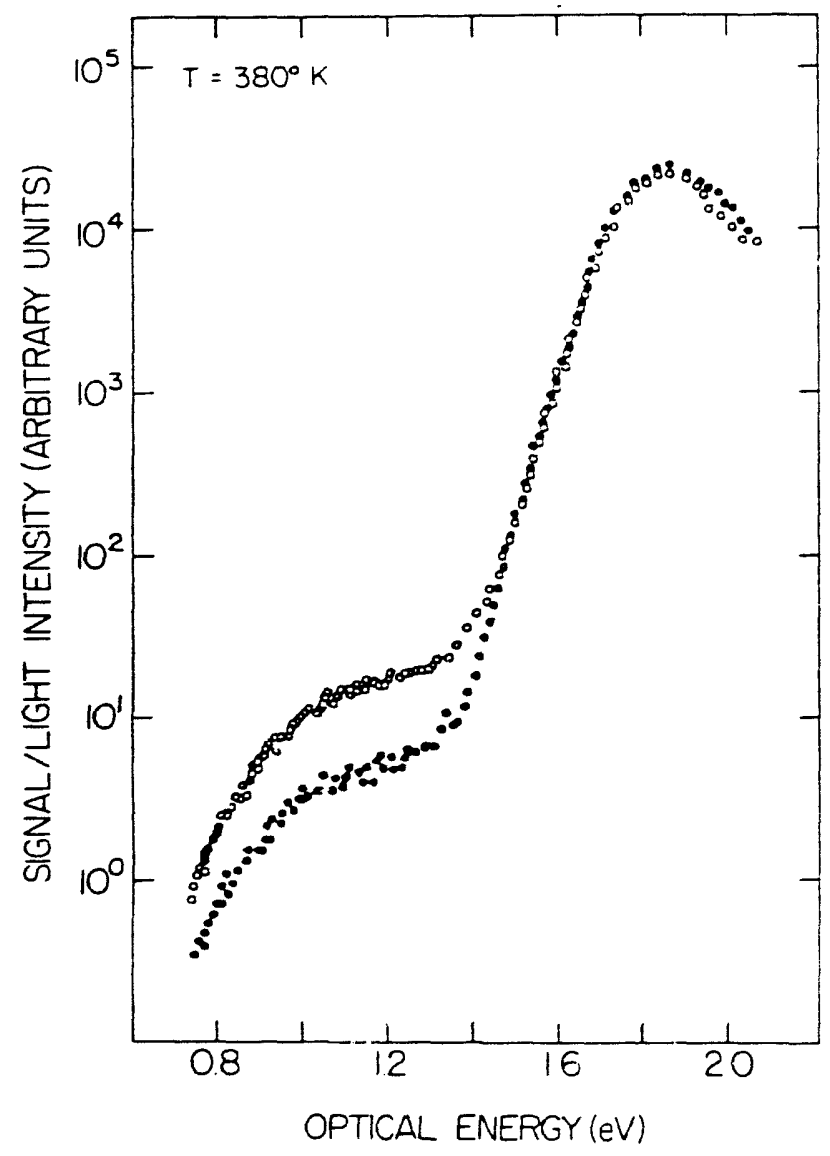


over a much larger energy range, particularly for undoped samples, but does not allow anabsolute determination of defect density or a simple determination of their spatial distribution.

The experimental details of our photocapacitance measurement method have been given previously. $[7,8]$ We can summarize its salient features as follows: A voltage pulse is used to fill gap states with electrons in the depletion region of the sample. Sub-bandgap light of a chosen optical energy is introduced after the voltage pulse to assist the re-equilibration of trapped carriers. However, we record every second transient without this sub-bandgap light so that a dark transient baseline can be established. The photocapacitance transient for a particular optical energy is the difference between the transients recurded in the light and dark. This transient is processed by multiplying it by a correlator function and integrating over time. This procedure establishes the optically induced variation in the junction capacitance, and hence the amount of optically released trapped charge, over a particular "time window" defined by the correlator function.

The photocapacitance spectrum is obtained by plotting the resultant, intensity normalized signal versus optical energy at constant temperature. Particular care is taken to operate at sufficiently low light levels that strict linearity to light intensity is observed.

These photocapacitance spectra appear qualitatively quite similar to subband-gap optical absorption spectra and are interpreted in a somewhat similar fashion. However, there are some advantages with this method useful for the current studies. Most important, perhaps, aside from the much greater sensitivity of this method compared absorption techniques, is that one is assured that the spatial region examined lies deep within the sample, roughly on the order of the depletion width (at least $3000 \AA$ from the junction interface). Thus we may experimentally determine the defect properties in the same region of the sample as that characterized by our profiling measurements.

Like optical absorption measurements, photocapacitance is relatively straightforward to interpret since it is not greatly affected by the details of the transport processes provided that measurement time scales are long compared to both dielectric relaxation times and carrier transit times across the depletion region. For intrinsic samples this sometimes requires us to operate at relatively low frequencies and elevated temperatures. However, for the studies of doped samples reported we may easily operate below room temperature at a 1 to 10 
$\mathrm{kHz}$ measurement frequency. We typically record capacitance transients over a timescale of 10 to $1000 \mathrm{~ms}$.

In Fig. 4 we display one pair of such photocapacitance spectra for a low defect undoped sample in state A and state B. These spectra clearly demonstrate the sensitivity of this method for identifying changes in the sub-band-gap absorption band related to the creation of metastable defects. (Additional examples of photocapacitance spectra will be given in Section 5).

\subsection{DEPLETION WIDTH MODULATED ESR SPECTROSCOPY}

The two most widely used methods to characterize defects in crystalline semiconductors have been electron spin resonance (ESR) and junction capacitan $=2$ methods, particularly capacitance DLTS. In a-Si:H the first method has been employed extensively to study the dominant deep defect (D-center) $[9,10]$ although it has been less useful in doped samples where defect states are empty or doubly occupied. In that case optical excitation can be employed to create some fraction of singly occupied, ESR visible center's [11,12]; however, total defect densities are then very difficult to estimate. For doped sarnples such densities are thus usually obtained from a number of other measurement techniques including sub-band-gap absorption or capacitance methods. However, one can then make only indirect arguments concerning the identification of such defects or their charge states. These ambiguities are compounded by the fact that defects near interfaces and in the bulk may have different properties or be in different charge states. Indeed, most measurement techniques, including standard ESR, have great difficulty distinguishing the source of the defects they observe.

In 1982 we performed the first experiments that combined the advantages of junction techniques with ESR spectroscopy through a method we termed "depletion width modulated ESR spectroscopy" (DWM-ESR). [13] Through this technique we were able to unambiguously assign the $\mathrm{D}^{-} / \mathrm{D}^{0}$ transition of the $\mathrm{g}=\mathbf{2 . 0 0 5 5}$ spin resonance signal to the peak in the density of states that was observed to lie near Ec $-0.9 \mathrm{eV}$ in n-type a-Si:H samples, as had been observed by DLTS [14] and optical absorption methods [15]. More recently we applied this method to assess the fundamental properties of deep defects in undoped a-Si:H. [16] 
Our measurments are carried using a standard Varian E-4 spectrometer with an X-band $(9.2 \mathrm{GHz}) \mathrm{TE}_{102}$ microwave cavity. We position a sample with a "large area" (roughly $0.5 \mathrm{~cm}^{2}$ ) Schottky barrier in the low E-field region of the cavity with electrical leads attached. Through these electrical connections we may then apply a DC and/or alternating voltage to vary the width of the depletion region in the sample. Typically we apply a square wave alternating voltage at a frequency from 1 to $5 \mathrm{~Hz}$ that switches between a high reverse bias of -8 or -6 volts to a low reverse bias of -1 or 0 volts. In this manner the depletion region width can be modulated by $0.2 \mu \mathrm{m}$ to as much as $1 \mu \mathrm{m}$.

We then employ lock-in detection of the reflected microwave signal at both the standard $100 \mathrm{kHz}$ magnetic field modulation and also at the low frequency of the bias modulation. The output from the low frequency lock-in is recorded both as an in-phase and quadrature signal vs. applied magnetic field. This produces a pair of derivative absorption ESR spectra entirely due to the changes in electronic occupation due to the changes in the applied junction bias. Because the volume of the modulated region inside the sample is quite small (on order $0.5 \mathrm{~cm}^{2} \times 3000 \AA$ ), many hours of signal averaging are required to obtain such spectra. However, background ESR signals (from sample surface contaminants, ravity walls, etc.) which are often the limiting factor in observing weak ESR signals, are completely absent.

In addition to standard ESR spectra plotted against applied magnetic field, we have also recorded spectra at fixed values of the DC magnetic field as a function of time. Such spectra enable us to examine more closely the precise relationship between voltage pulse filling and thermal escape of carriers from deep defects and their changes of spin state.

Such spin signals may be calibrated in absolute terms. The most reliable standards were obtained using a series of phosphorus doped crystalline $\mathrm{Si}$ samples whose dopant levels were measured directly. [16] At low temperatures the characteristic signal of electrons bound to these donor levels was easily determined measured using the same sample probe assembly as for our a-Si:H samples. We took into account a variety of factors including the differences in ESR lineshapes, cavity Q's, spin polarization factors, etc., to arrive at our stated absolute spin densities.

These spin signals may then be directly compared with charge signals, also calibrated as absolute densities, determined by capacitance transient signals on the same samples under exactly the same measurment conditions. In this 
fashion we may unambiguously identify the nature of the deep state transitions that are being observed. We may also be quite certain that our signals arise from a spatial region deep within the bulk of each sample.

\subsection{LIGHT-INDUCED DEFECTS IN SAMPLES WITH MODULATED CARBON IMPURITIES}

\subsection{BACKGROUND}

Several early studies indicated significant carbon and oxygen impurity components to the light induced degradation of intrinsic a-Si:H samples and solar cells. [1,2] However, more recent studies using ESR or the PDS optical absorption method to monitor the changes in the deep defect density of states tended to rule out a role of atmospheric impurities. $[3,4,17]$ Because these studies utilized samples grown in UHV systems, in which an order of magnitude smaller concentration of impurities is obtained than in conventional glowdischarge systems, this work seemed fairly definitive. Indeed, these samples still showed similar levels of degradation to conventionally grown samples. Moreover, the degradation was found to be independent of impurity content at levels below 1 at\%.

- Our own junction capacitance based measurements of light-induced deep defect creation had nonetheless strongly suggested larger degrees of degradation for samples with larger concentrations of carbon impurities in the trace concentration regime (below 0.1at.\%). [6] Thus we decided to pursue this possible connection with extrinsic carbon levels somewhat further. To be sure that we could isolate the effects due to specific impurities from other changes in the growth process that occur between successive runs, we utilized samples whose impurity content was intentionally modulated during growth (see Section 2). We then exploited the unique ability of junction capacitance methods to examine the spatial dependence of the metastable induced defects within a single sample.

\subsection{EXPERIMENTAL RESULTS}

Figure 5(b) shows typical data obtained from drive-level capacitance profiling (DLCP). The SIMS impurity profiles for this sample are shown in Fig. 5(a). Unlike a sample with uniform spatial properties that should exhibit a flat 

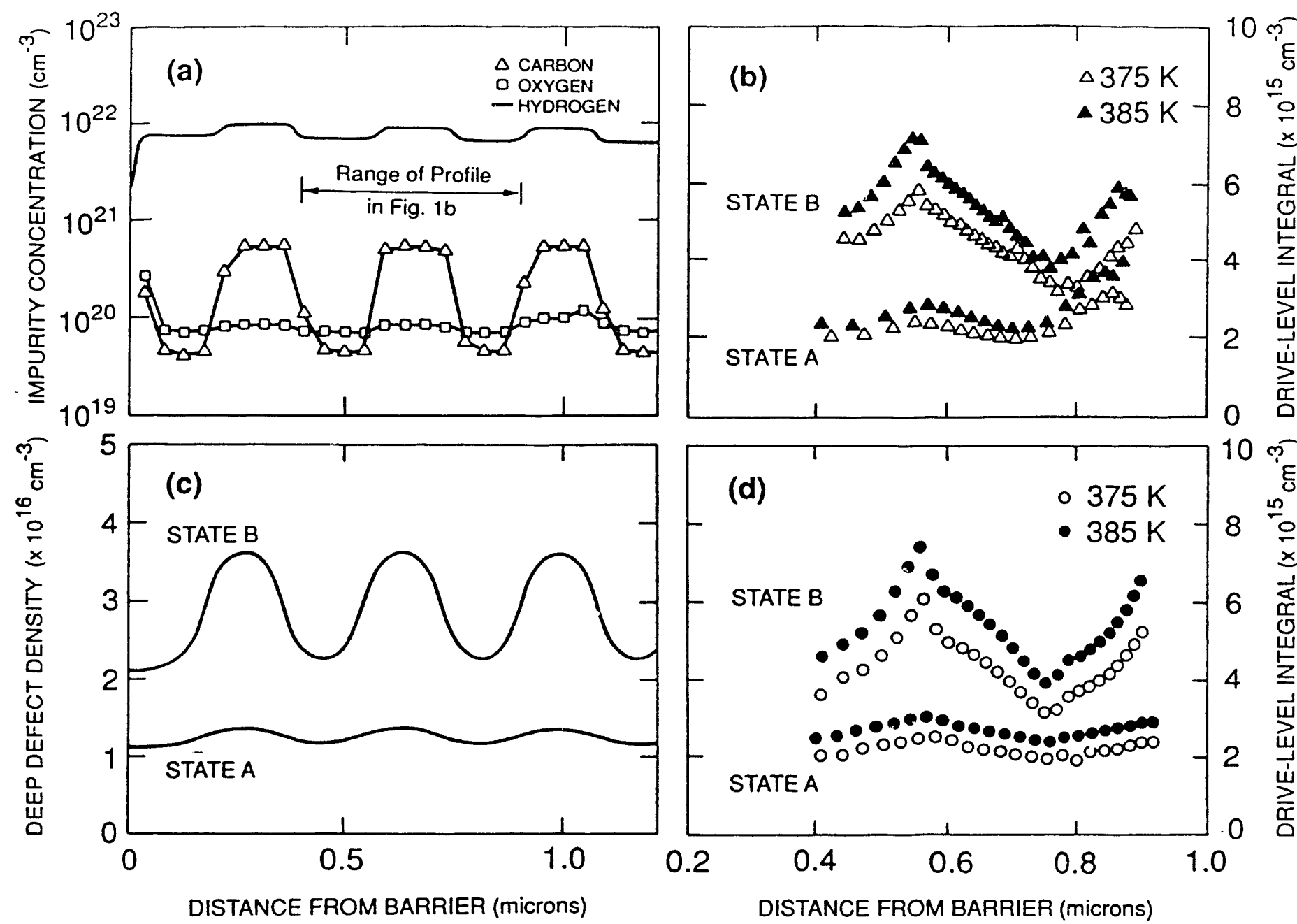

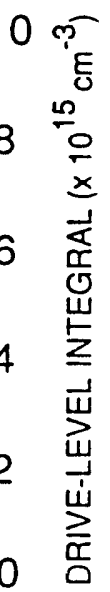

FIG. 5. (a) SIMS deduced profiles for $\mathrm{C}, \mathrm{H}$, and $\mathrm{O}$ in sample showing the spatially varying carbon concentrations introduced by modulating the $\mathrm{CH}_{4}$ gas content during growth.

(b) DLCP data indicating the spatial variation of the deep defect derisities for states $A$ and $B$ at two temperatures. These profiles were obtained by varying the $\mathrm{DC}$ bias between $-6 \mathrm{~V}$ and $-1 \mathrm{~V}$.

(c) Spatial variation of the total deep density in states a and b as deduced by numerical modeling of the DLCP shown in (b).

(d) Calculated DLCP curves using the spatial variation of deep defects as shown in $(\mathrm{c})$ 
DLCP behavior (for example, see Fig. 3), the profiles for this sample in both states $A$ and $B$ show a periodic variation that exactly matches the period of the carbon profile in Fig. 5(a). The amplitude of this variation in state B, however, is much larger than for state A. This indicates a greater increase in defects after light soaking in the carbon-rich regions than in the carbon-poor regions. Also, while the curves in Fig. 5(b) appear sawtooth-like in contrast to the more squarewave appearance of the SIMS impurity profile, this is actually an artifact due to the finite spatial resulution of the DLCP method. This tends to average the density of states over a distance scale roughly equal to the Debye length, or about $1000 \AA$ in this sample.

Figure 5(b) displays DLCP curves at two different measurement temperatures for each metastable state of the sample. For samples that exhibit only a small spatial variation one could obtain $g(E)$ as a function of position simply by subtracting the curves taken at these two temperature and then dividing by the corresponding thermal energy differences as given by Eq. (3). This would give $g(E, x)$ at an energy depth corresponding to a frequency of $100 \mathrm{~Hz}$ at $380 \mathrm{~K}$; approximately $0.8 \mathrm{eV}$ below $\mathrm{E}_{\mathrm{C}}$. Since this energy regime lies roughly in the middle of the $D$ defect band, we would thus obtain a spatial profile of the defect concentration within the sample.

- The spatial variation within this sample is much larger than that allowed by such a simple analysis. Thus, to properly understand these results, we require a full numerical simulation of the DLCP data based on an assumed function $g(E, x)$. If we are then able to reproduce our observed profiles at each temperature we can be fairly confident that we have chosen the correct $g(E, x)$ to represent our sample.

The results of this simulation are displayed in Fig. 5(d) based on the assumed defect spatial variation shown in Fig. 5(c). We are indeed able to reproduce our experimental data fairly well and thus obtain the result that deep defect densities are indeed much more greatly increased in the carbon rich regions compared to the carbon poor regions after light soaking (by nearly a factor of 2). Additional information is obtained concerning the distance scale of the transition between these two regions. We discuss our modeling procedure and results in more detail in the next section. 


\subsection{NUMERICAL MODELING OF DRIVE-LEVEL PROFILES}

We developed methods to numerically model the drive-level profiling data, assuming a density of states in each region. We assume a Gaussian shaped deep defect band located at $0.9 \mathrm{eV}$ below $\mathrm{E}_{\mathrm{C}}$ with a full width at half-maximum of $0.3 \mathrm{eV}$. This assumed defect band agrees with the position and width obtained by sub-band-gap optical specta [18].

A total of 4 parameters were varied to obtain the fits to our DLCP data for each state. Two of these were the magnitudes of the deep defects in the carbonrich and carbon-poor regions. The third was the position of the neutral bulk Fermi level in the sample. This parameter was somewhat constrained from the measured value of activation energy, $E_{\sigma}$, of the dark conductivity of the sample (which should roughly have the value $E_{C}-E_{F}$ ). We also assumed that the neutral Fermi level position relative to $E_{C}$ would be the same in the two regions. This last assumption would not be valid for the case of dopant impurities. However, we determine that $\mathrm{E}_{\mathrm{F}}{ }^{0}$ could vary by at most $\pm 10 \mathrm{rneV}$ between the two regions to be consistent with the tempurature dependence of the profiles that we observe.

The final parameter required to obtain good fits to the experimental profiles controlled the degree of abruptness with which the electronic properties changes between the two spatial regions. This parameter was expressed as a characteristic decay length, $\xi$, such that the defect density of states in region "a" at a distance $\Delta x$ from the boundary with region " $b$ " should be given by

$$
g_{a}(E, x)=\frac{1}{2} \exp (-\Delta x / \xi) g_{b}(E)+\left[1-\frac{1}{2} \exp (-\Delta x / \xi)\right] g_{a}(E)
$$

Here $g_{a}(E)$ and $g_{b}(E)$ represent the densities of states for the two regions far from any boundaries.

Figure 6 illustrates a series of calculations in whcih the value of $\xi$ is varied between $125 \AA$ and $500 \AA$. Other parameters have been chosen to be consistent with the defect parameters measured for the sample data of Fig. 5. We see that the shape of the drive-level profiles is quite sensitive to the degree of sharpness at the boundary and that, for $\xi$ near $400 \AA$, we obtain the observed sawtooth shape of the experimental profiles fairly accurately. 


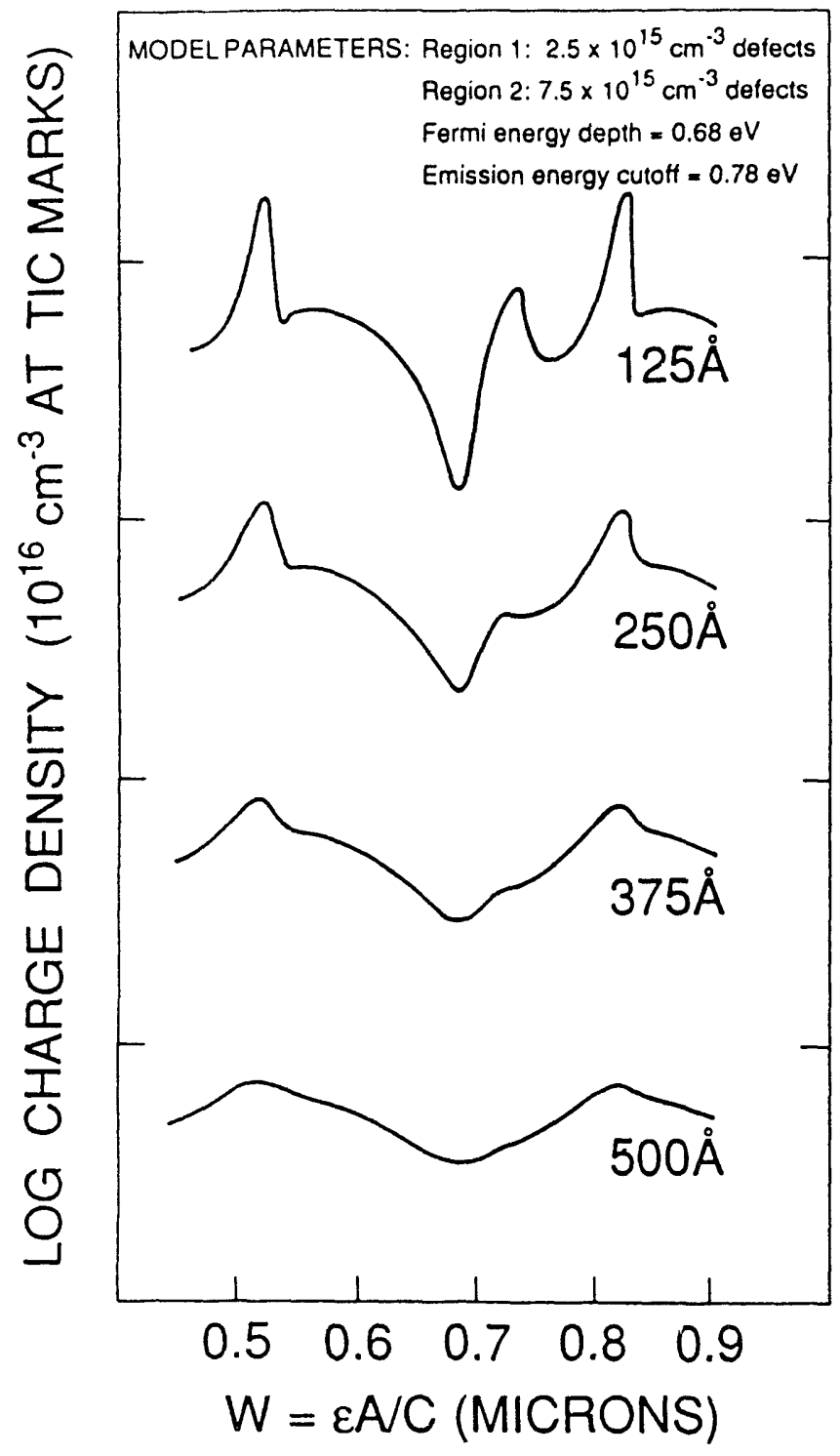

FIG.6. A series of calculated drive-level profiles for different values of a parameter $\xi$ which determines the abruptness of the change of electronic properties at the boundary between the high and low defect regions. Other parameters used in the calculation are also given. 


\subsection{DISCUSSION}

As previously noted, our analysis for the data in Fig. 5 indicates that lightsoaking results in almost twice as many defects in the carbon-rich regions compared to the carbon-poor regions, thus indicating a significantly higher degradation for those regions. Our modeling also suggests that the change in electronic properties at the interface between the different regions was generally more abrupt in state $B$ than in state $A$. We obtained a value of $\xi$ of $400 \AA \pm 50 \AA$ in state $B$ as compared to $650 \AA \pm 100 \AA$ in state $A$. This smoother transition for state A seems consistent with the notion that dark annealing results in a higher degree of equilibration between the two regions.

Figure 8(a) summarizes the variation in defect densities as a function of carbon content before and after light-soaking for several samples. For state A and we observe that the defect density increases slightly with impurity content. However, the larger slope in state B indicates the significantly larger degradation for regions of higher carbon content. This increase actually appears sub-linear with carbon ccencentration such that an increase in concentration by one ord $=1$ of magnitude results in perhaps 2 to 3 times as many light induced defects. However, even at levels as low as 0.1 at.\% the spatial variations in increased metasstable defect densities are unmistakable in the actual profiles. (Fig. 7 displays results for a sample with one of the lowest carbon impurity modulation profiles.) We must therefore conclude that a strong correlation exists between light-induced degradation and the carbon concentrations at levels between 0.1 and 1 at $\%$.

It has been suggested that the our observed increased degradation may instead be due to the enhanced hydrogen content in the carbon-rich regions [Figs. 1 and 5(a)] caused by the methane admixture. In Fig. 8(b) we therefore plot the defect densities for the same samples as a function of the SIMS determined hydrogen content. In this case there appears to be no significant correlation between total hydrogen content and light-induced metastable degradation in our samples.

It has also been suggested that the addition of even such small quantities of carbon may significantly increase the number of weak bonds and thus indirectly lead to an increased susceptibility for degradation. The Urbach energy arameter $E_{u}$, indicating the width of the valence bandtail in a-Si:H, has 


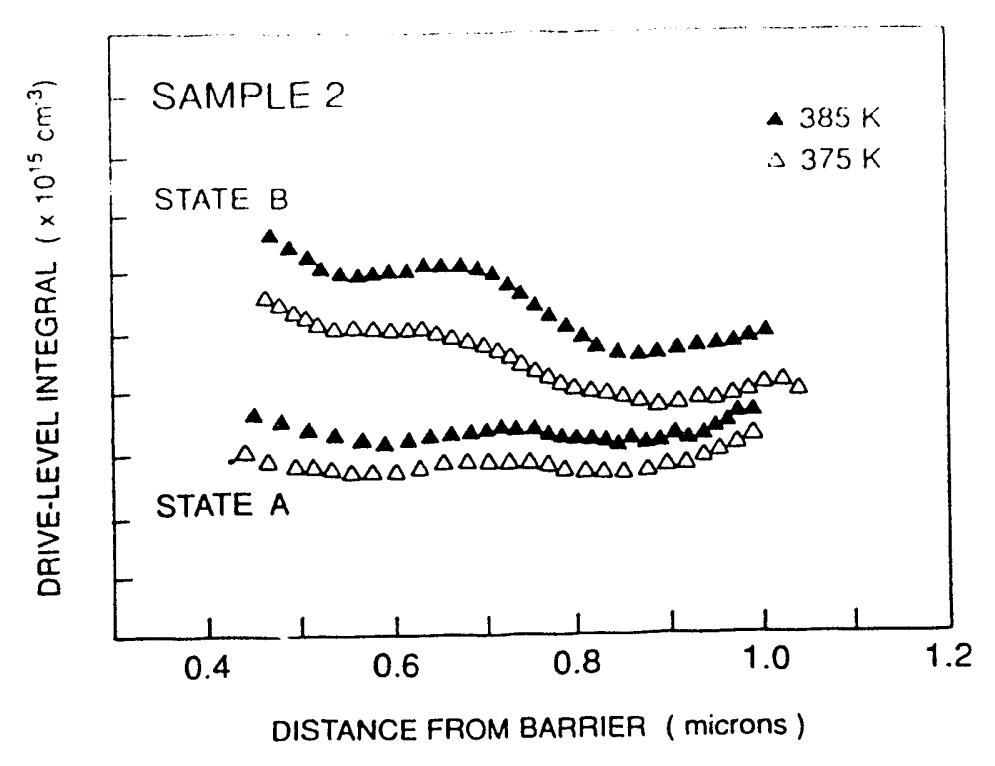

FIG. 7. Drive-level profiling data for a low carbon modulated sample at two different temperatures for states $\mathrm{A}$ and $\mathrm{B}$. The carbon content in this sample varied between 0.1 at.\% and 0.25 at.\%.
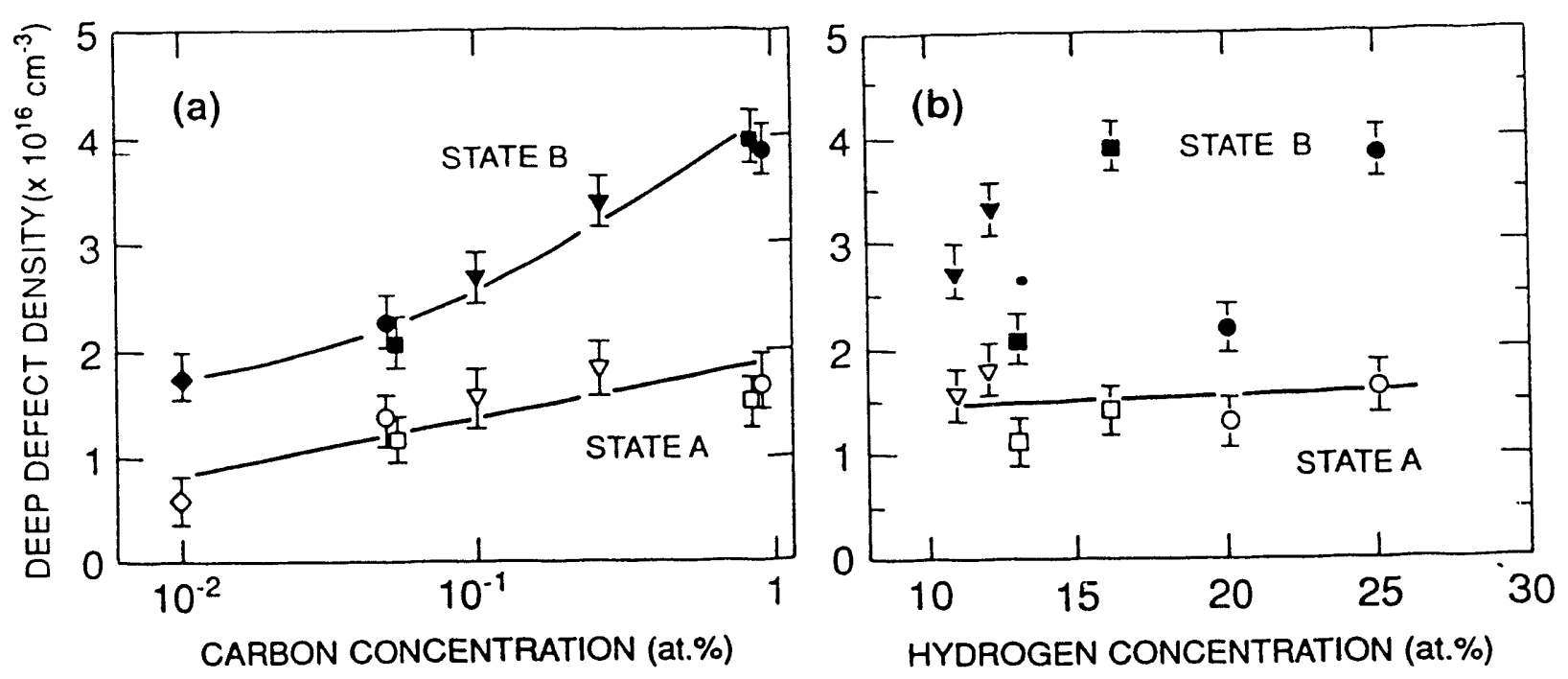

FIG. 8. (a) Total deep defect densities in state A and B deduced for several films as a function of carbon content. For each spatially modulated film the defect density is given for both the carbon-rich and the carbon-poor regions using an identical symbol. The data points $($,$) for the lowest carbon content were$ obtained from a uniform sample.

(b) Defect densities as in Fig. 8(a), however now displayed as a function of total hydrogen content. Identical symbols again indicate different spatial regions within one sample. 
generally been linked to this kind of disorder. [19] Indeed, it is fairly wellknown that a-Si:H films containing impurities at levels greater than 5 at $\%$ do exhibit much broader band tails $\left(E_{\mathbf{u}}>60 \mathrm{meV}\right)$ than high-quality intrinsic a-Si:H films $\left(E_{\mathbf{u}} \sim 40-50 \mathrm{meV}\right)$. [20] We thus have determined the characteristic bandtail energies of our films with transient photocurrent methods. However, we found that at levels of up to 1at.\% impurities our samples did not indicate a significant increase in $E_{u}$ values with increasing impurity content. Rather the Urbach energies were all found to lie close to $53 \mathrm{meV}$. This tends to rule out any major differences in the concentrations of weak bonding sites between the regions of low and high carbon content in our samples.

Our results stand in sharp contrast to earlier conclusions based on PDS and ESR measurements requiring impurity concentrations of more than 5at.\% before any such changes in metastable behavior became significant. [3,4,17] We believe that this enhancement occurs because of local changes in the environment surrounding deep defect sites. Impurities generally add more configurational degrees of freedom to the amorphous network and therefore are likely to increase the number of possible routes to metastable defect formation. Altered bonding environment due to, say, C-Si, $\mathrm{C}-\mathrm{C}$ and $\mathrm{C}-\mathrm{H}$ bonds in place of $\mathrm{Si}-\mathrm{Si}$ or $\mathrm{Si}-\mathrm{H}$ bonds undoubtedly will modify the energy barriers between local metastable configurations. This may readily result in an increased susceptibility to light-induced degradation.

\subsection{INVESTIGATION OF DEFECT REACTIONS IN PHOSPHORUS DOPED SAMPLES}

\subsection{BACKGROUND}

Metastable states can be studied to provide clues concerning microscopic defect reactions in a-Si:H. In the popular model for light-induced defects, the recombination of photoinduced carrier breaks weak $\mathrm{Si}-\mathrm{Si}$ bonds and creates deep defect states which are dangling bonds. [9] In n-type doped samples these will be produced in a negative charge state $\left(\mathrm{Si}_{3}{ }^{-}\right)$:

$$
2 \mathrm{e}^{-}+2 \mathrm{Si}_{4}{ }^{0} \Longrightarrow 2 \mathrm{Si}_{3}^{-}
$$

However, in phosphorus doped samples there have been proposed extensions of this reaction that include bond switching with dopant atoms $[6,21,22]$ : 


$$
\begin{aligned}
& \mathrm{Si}_{4}{ }^{0}+\mathrm{P}_{3}{ }^{0} \Longleftrightarrow \mathrm{Si}_{3}^{-}+\mathrm{P}_{4}^{+} \\
& \mathrm{Si}_{4}{ }^{0}+\mathrm{P}_{4}^{+}+2 \mathrm{e}^{-} \Longleftrightarrow \mathrm{Si}_{3}^{-}+\mathrm{P}_{3}{ }^{0-}
\end{aligned}
$$

All of the above proposed reactions involve a change in density of dangling bonds which, because we are considering n-type doped material, appear in a negative charge state $\left(\mathrm{Si}_{3}{ }^{-}\right)$. The presence (or absence) of extra electrons on one side of the reaction implies a shift of the Fermi level $\left(E_{F}\right)$ to change the occupation of states. One thus notes that, for the 3 reactions listed above, that there is a distinct difference in the ratio of dangling bonds created to the number of electrons transferred from the Fermi energy.

It should be clear from the above remarks that a quantitative comparison between the changes in occupation of states near $E_{F}$ and the density of deep defects could be used to identify which of these reactions dominates a given change in metastable state. On the basis of some preliminary studies along these lines a few years ago [23] we suggested that one must include reactions that would be able to activate or deactivate a dopant atom without changing the density of dangling bonds, $N_{D}$. That is, we proposed a half reaction of the form:

$$
\mathrm{P}_{3} \mathrm{O}^{\Longleftrightarrow} \mathrm{P}_{4}^{+}+\mathrm{e}^{-}
$$

As part of our SERI subcontract we have now examined the evidence for such a dopañt activation mechanism in more detail.

\subsection{EXPERIMENTAL RESULTS}

We investigated the changes in deep defects, $N_{D}$, and in the number of occupied bandtail states, $\mathrm{N}_{\mathrm{BT}}$, for a variety of metastable states produced by light-soaking, the partial annealling of light soaked states, and quench cooling. The procedure for producing these states are given in Section 2. We then utilized drive-level capacitance profiling at lower temperatures (typically $240 \mathrm{~K}$ for a measurement at a $10 \mathrm{kHz}$ frequency) to determine $\mathrm{N}_{\mathrm{BT}}$ for each of these metastable states. Transient photocapacitance spectroscopy was employed to compare the changes in deep defects. Drive-level profiling results at higher temperatures were used to help calibrate these changes as absolute densities. The detailed procedure for carrying out these measurements have been given in Section 3. 


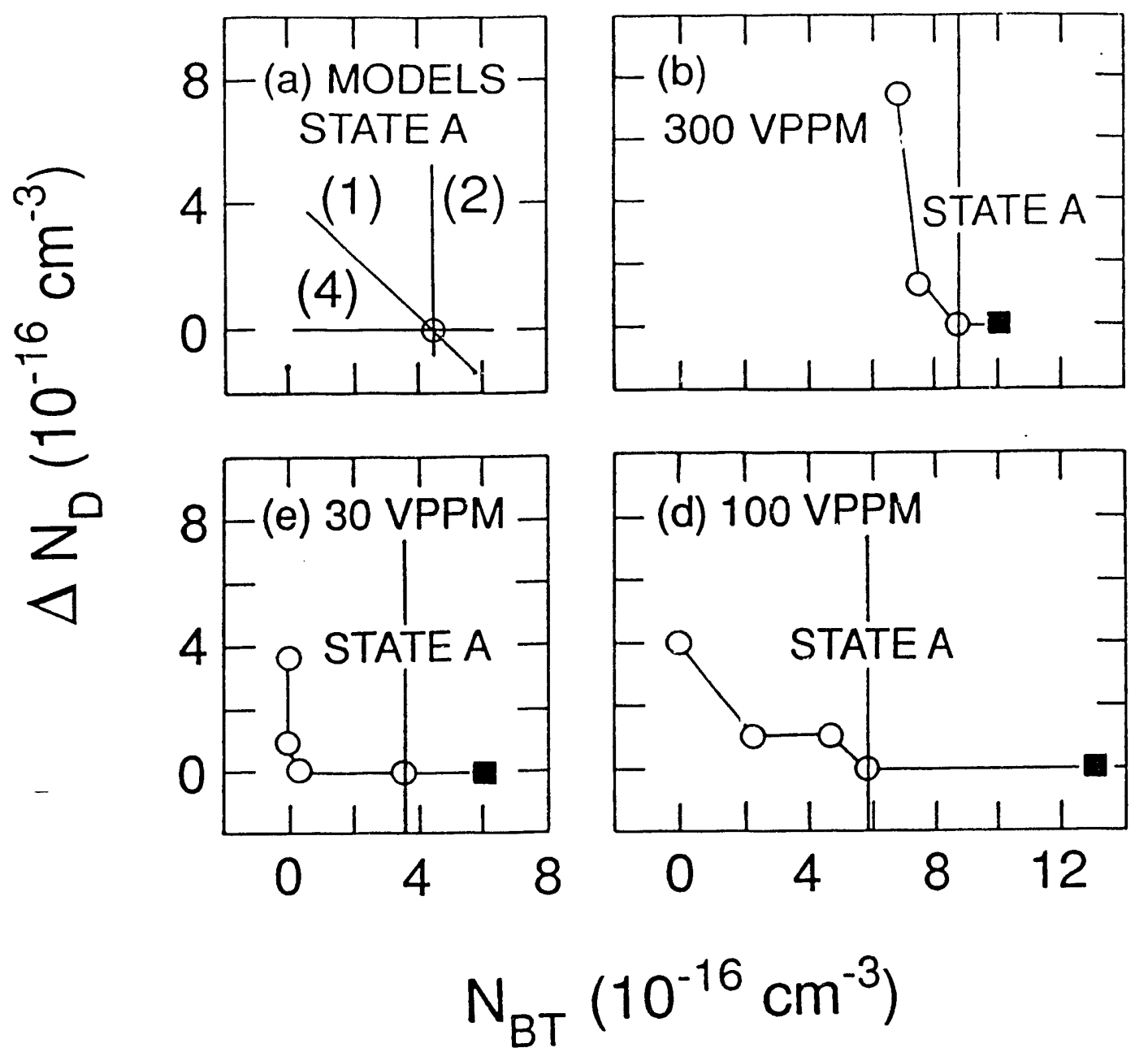

FIG. 9. Change in the deep defect densities $\left(N_{D}\right)$ as a function of the occupied bandtail state density $\left(\mathrm{N}_{\mathrm{BT}}\right)$ for (a) the proposed defect reations (1), (2), and (4) discussed in the text; (b) the $300 \mathrm{Vppm} \mathrm{PH}_{3}$ doped sample; (c) the $30 \mathrm{Vppm} \mathrm{PH}_{3}$ doped sample; and (d) the $100 \mathrm{Vppm} \mathrm{PH}_{3}$ doped sample. Open circles denote metastable states obtained by light-soaking and partial annealing, and the filled squares indicate quench-cooled metastable states. The vertical lines denote the $\mathrm{N}_{\mathrm{BT}}$ values of the sample in state A. Error bars are comparable to the data point sizes. 
Our results for the changes in $\mathrm{N}_{\mathrm{BT}}$ and $\mathrm{N}_{\mathrm{D}}$ with metastable treatment for our 3 most heavily doped samples have been plotted in Fig. 9 in a manner which can be compared directly with the predictions of the proposed defect reaction models (Eqs. I-IV). The horizontal position indicates the DLCP determined value of $N_{B T}$ with a vertical line indicating its value in state $A$. The vertical axis shows the deviation in the value of $N_{D}$ from its state $A$ value and is plotted in the same density units as $\mathrm{N}_{\mathrm{BT}}$. A zero value for $\mathrm{N}_{\mathrm{BT}}$ signifies that $\mathrm{E}_{\mathrm{F}}$ is deeper than $0.4 \mathrm{eV}$ below $\mathrm{E}_{\mathrm{C}}$ so that there are no occupied conduction bandtail states. Open circles denote metastable states obtained by light-soaking and partial annealing; the filled squares denote the quench-cooled metastable states.

We observe that many metastable treatments lead to a change in $\mathrm{N}_{B T}$ without a change of comparable magnitude in $N_{D}$. This is, in fact, the case for all of the quench-cooled metastable states. A representative comparison of the actual photocapacitance spectra indicating the changes in $N_{D}$ for sorne of these metastable states is given for one sample in Fig. 10.

In Fig. 11 we summarize the total $\mathrm{N}_{\mathrm{D}}$ vs. $\mathrm{PH}_{3}$ doping level for the fully light-soaked (state B) and state A measurements for 5 samples that have been fully characterized in this fashion. We note that the relative increase in deep states after light-soaking approaches a factor of 2 for the most lightly doped samples but that this ratio decreases as the doping level increases. We also plot $\mathrm{N}_{\mathrm{BT}}$ vs. doping level and compare the values of $\mathrm{N}_{\mathrm{BT}}$ between the slow-cooled states (state $\mathrm{A}$ ) and the quench-cooled metastable states. Here we also obser e a factor of two increase in $\mathrm{N}_{\mathrm{BT}}$ in many of the samples studied.

The straight lines included in this figure are for comparison purposes to indicate the approximate power law dependence of $\mathrm{N}_{\mathrm{D}}$ and $\mathrm{N}_{\mathrm{BT}}$ on $\mathrm{PH}_{3}$ doping level. These lines have slopes of 1.0 and 1.5 , respectively.

\subsection{DISCUSSION}

The correlations between $\mathrm{N}_{B T}$ and $\mathrm{N}_{D}$ vary markedly among the proposed defect reactions. The predictions resulting from reactions (I), (II), and (IV) are indicated schematically in Fig. 9(a). Only the most heavily doped sample appears to possibly be consistent with a reaction of type (II). That reaction maintains a balance between the densities of $\mathrm{P}_{4}{ }^{+}$and $\mathrm{Si}_{3}{ }^{-}$so that one could expect significant changes in $N_{D}$ with very little shift in. $E_{F}$ (and hence little change in $\mathrm{N}_{\mathrm{BT}}$ ). In general, hơwever, the metastable changes generated by light 


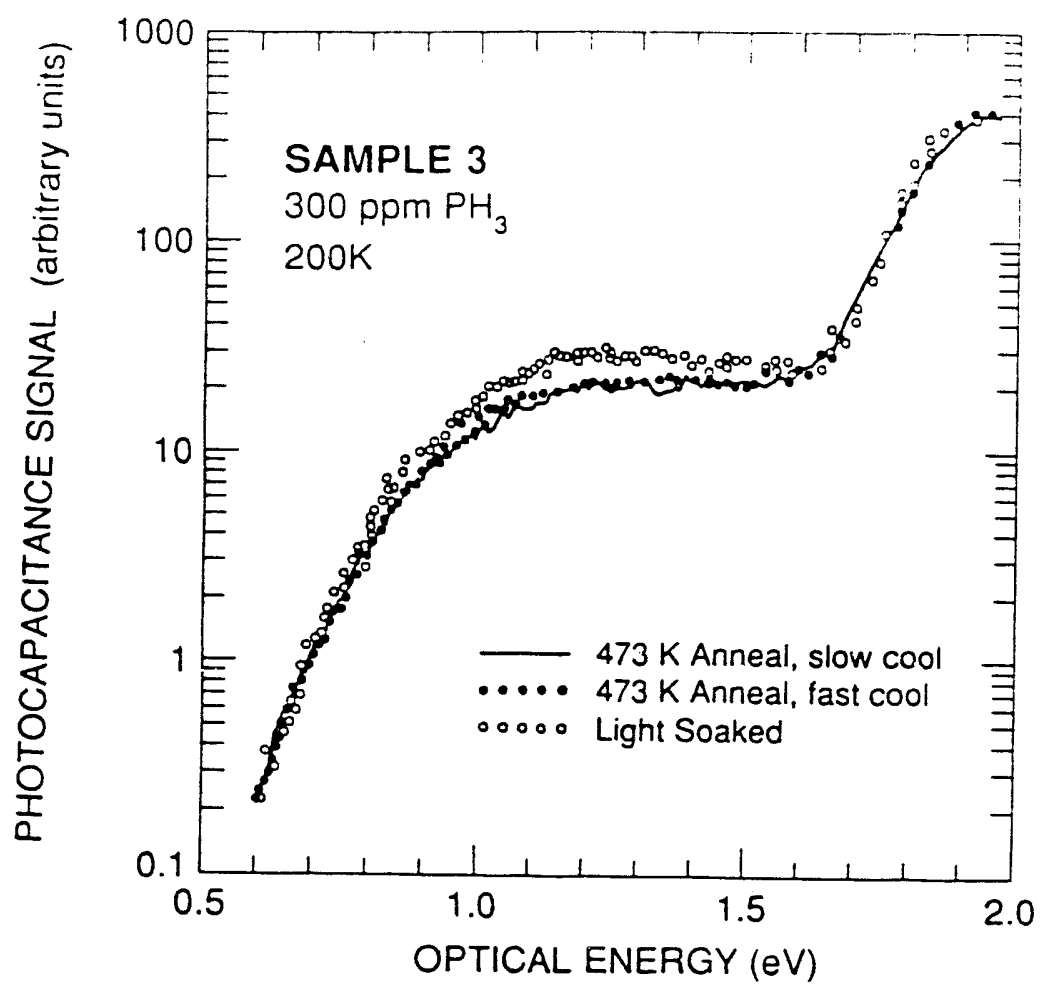

FIG. 10. Voltage pulse transient photocapacitance spectra taken for a $300 \mathrm{Vppm}$ $\mathrm{PH}_{3}$ coped sample for state $\mathrm{A}$, and also for states following light-soaking and following quench cooling.

FIG. 11. Summary of the total deep defect densities $\left(N_{D}\right)$ vs. $\mathrm{PH}_{3}$ doping level for the fully light-soaked state (state B) and state A measurements. Also plotted are the values of conduction bandtail occupation $\left(\mathrm{N}_{\mathrm{BT}}\right)$ vs. doping level for state $\mathrm{A}$ and the quench-cooled states. The straight lines indicate the approximate power law dependence of $N_{D}$ and $N_{B T}$ for state $A$ on $\mathrm{PH}_{3}$ doping level and have slopes of 1.0 and 1.5 , respectively.

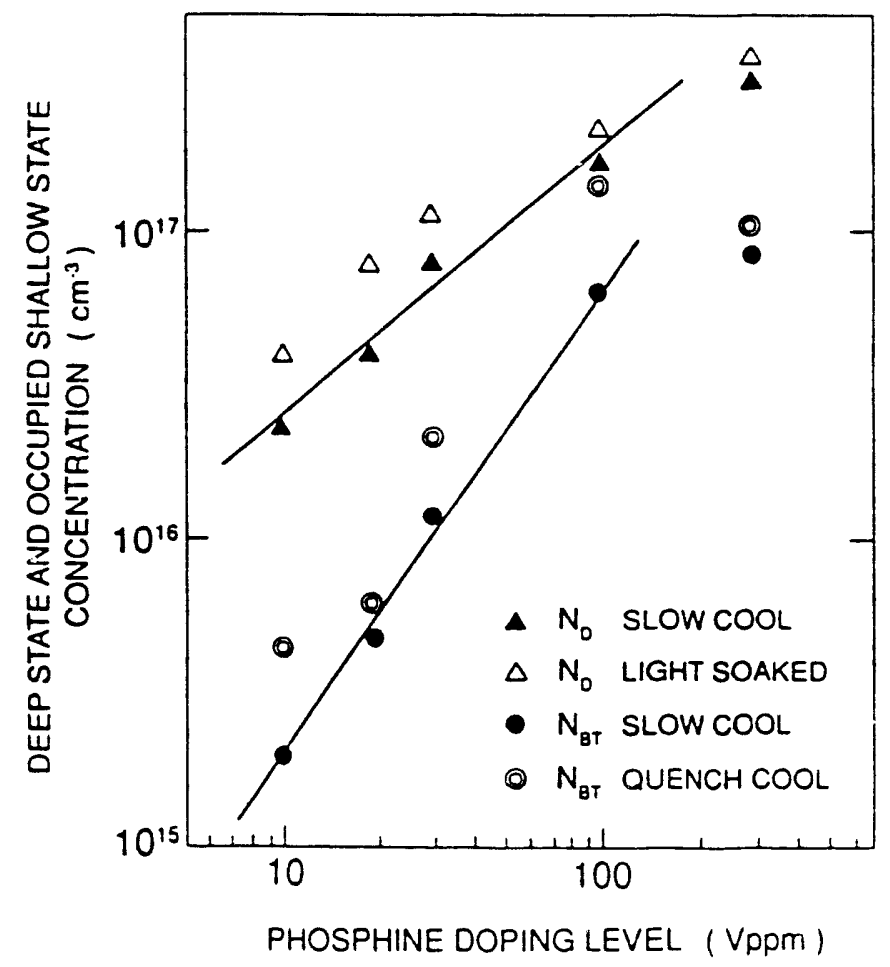


soaking and partial annealing do not seem to obey reactions of strictly a single type.

On the other hand, there is very consistent behavior for the change in properties associated with quench cooling. Here we observe large changes in $\mathrm{N}_{\mathrm{BT}}$ without any observable change in $\mathrm{N}_{\mathrm{D}}$. This result appears to contrast markedly with reported results for the quench cooling of intrinsic a-Si:H samples. [24] Such a result can only be accounted for by a process like reaction (IV) in which phosphorus changes its doping coordination independent of bond switching with silicon atoms. Although one might attempt a composition of reactions (if types (I) and (II) to accomplish the same net result as reaction (IV), we do not believe this a reasonable alternative because it would require that a second dangling bond be fortuitously located very close to the $\mathrm{Si}_{3}{ }^{-} / \mathrm{P}_{4}{ }^{+}$pair created by reaction (II), a situation that must be considered extremely unlikely.

There has been a great deal of discussion of hydrogen diffusion mechanisms accounting for metastable behavior in a-Si:H, and we recently proposed a couple hydrogen bond switching reactions which could result in the activation of a donor without changes in $\mathrm{N}_{\mathrm{D}}:[25]$

$$
\begin{aligned}
& \mathrm{P}_{3}{ }^{0}+\mathrm{Si}: \mathrm{H} \Longleftrightarrow \mathrm{P}_{4}^{+}+\mathrm{Si}_{4}{ }^{0}+\mathrm{e}^{-}+\mathrm{H}^{*} \\
& \mathrm{P}: \mathrm{H}+\mathrm{Si}_{4}{ }^{0} \Longleftrightarrow \mathrm{P}_{4}^{+}+\mathrm{Si}: \mathrm{H}+\mathrm{e}^{-}
\end{aligned}
$$

where $: H$ represents a passivated bond on an otherwise 3-fold coordinated atom and $\mathrm{H}^{*}$ is an interstitial hydrogen. Such proposed reaction are also quite consistent with recent studies of hydrogen in crystalline silicon where evidence for a mobile species of negatively charged hydrogen has been found. [26] These researchers have proposed the reaction

$$
(\mathrm{P}-\mathrm{H})^{0} \Longleftrightarrow \mathrm{P}_{4}^{+}+\mathrm{H}^{-}
$$

where $(\mathrm{P}-\mathrm{H})^{0}$ is a neutral hydrogen-dopant complex. In a-Si:H, the existence of such a reaction could quite naturally account for metastable changes observed in n-type material a-Si:H under bias annealing [27] where $\mathrm{N}_{B T}$ has been observed to increase dramatically while, as our more recent studies of this effect have shown, there is no observable change in $N_{D}$.

The observations of the increased doping effects of reaction (VII) have been made through the imposition of an applied electric field to remove the $\mathrm{H}^{-}$ species from one region of the sample. [26] However, if one speculates that reaction (VII) might be followed by the ultimate conversion of $\mathrm{H}^{-}$into a neutral species, this could lead to an increase in $\mathrm{N}_{\mathrm{BT}}$ in a manner functionally equivalent to that given in reaction $(\mathrm{V})$. 
Finally, we should comment on the nearly linear dependence of $N_{D}$ on - phosphorus doping suggested in Fig. 11. Previously it has been argued that $N_{D}$ scales as $[\mathrm{P}]^{1 / 2}$. [28] Because our only knowledge of the phosphorus content in these samples is based on gas phase concentrations, it is not entirely certain that we have a linear dependence on actual phosphorus incorporation. However, it has been found elsewhere that $[\mathrm{P}]$ generally does scale linearly with $\mathrm{PH}_{3}$ levels present during growth. [29] Also, our own data suggests the usual sublinear dependence of $\mathrm{N}_{\mathrm{D}}$ with $[\mathrm{P}]$ at levels above $100 \mathrm{Vppm} \mathrm{PH}_{3}$.

$\mathrm{Tl}$ :soretically, one understands the $1 / 2$ power law dependence as a consequence of reaction (II) in the equilibration process of dangling bonds together with the requirements of charge neutrality so that $\left[\mathrm{P}_{4}^{+}\right] \cong\left[\mathrm{N}_{\mathrm{D}}{ }^{-}\right]$. However, given that alternative defect reactions may actually dominate metastable processes for activating $\mathrm{P}_{4}$ dopants and that an $\mathrm{H}^{-}$species could alter the charge balance condition, it is not unreasonable to speculate that a linear dependence of $\mathrm{N}_{D}{ }^{-}$on [P] could occur in the more lightly doped samples.

A simpler explanation might be that silicon-dopant complexes actually form in the plasma-phase during film growth and that these dissociate upon deposition but do not react in solid-phase thermal cycling. This would result in a linear dependence of $\mathrm{N}_{D}$ on the gas phase $\mathrm{PH}_{3}$ concentration and still provide a mechanism for self-compensation. Within such a picture phosphorous dopants and deep defects would each equilibrate independently (as our results indicate); however, the two processes would often be correlated due to the effect the Fermi level position has on each of them. Indeed, convincing evidence that deep defect formation responds to $\mathrm{E}_{\mathrm{F}}$ position independent of any dopant bond switching reaction has recently been obtained through post-growth interstitial lithium doping. [30]

Finally, it has also been suggested that dopant impurities account directly for both stable and light-induced deep defects through the formation of dopant complexes similar to the DX center in III-V semiconductors. [31] Such a model would quite naturally lead to the observed linear dependence of deep defect with dopants in the low concentration regime.

The correct explanation will hopefully also ultimately account for the observed $3 / 2$ power law dependence of $\mathrm{N}_{\mathrm{BT}}$ on $\mathrm{PH}_{3}$ doping. Indeed, the previously proposed explanations for a $1 / 2$ power law of $N_{D}$ on doping also lead to a $1 / 2$ power for $N_{B T}$, which is totally inconsistent with our own data and 
the previous studies which obtained $\mathrm{N}_{\mathrm{BT}}$ using the carrier sweep-out technique. [28]

\subsection{SPIN AND CHARGE STATES ASSOCIATED WITH METASTABLE DEFECTS IN PHOSPHORUS DOPED SAMPLES}

\subsection{BACKGROUND}

It is generally believed that the $D$ defect is associated with the isolated silicon dangling bond which in intrinsic material is singly occupied and gives rise to the characteristic ESR signal with $g=2.0055$ [9]. For phosphorus doped samples this defect is doubly occupied and thus diamagnetic. However, the same characteristic ESR signal may be observed by optical excitation in such samples [11], and it may also be observed in the dark by removing some of the defect electrons in the depletion region of a Schottky barrier or diode junction. This latter technique is termed "depletion width modulated ESR" (DWM-ESR) spectroscopy. The experimental details of this method have been described in Section 3.

The DWM-ESR technique was first applied to study deep defects in phosphorus doped a-Si:H where it was possible to identify the $\mathrm{D}^{-} / \mathrm{D}^{\circ}$ transition from $a$ band near $E_{C}-0.9 \mathrm{eV}$. [13] These experiments also confirmed that the effective correlation energy, $U_{\text {eff }}$, for this defect band had to be significantly position (at least $0.2 \mathrm{eV}$ ). Strikingly different results were obtained very recently on intrinsic a-Si:H where it appeared that $U_{\text {eff }} \equiv 0$. [16] These latter results seemed to be independent whether the sample was in state A or state B.

The initial DWM-ESR experiments on n-type a-Si:H were carried out on fairly heavily doped samples (100 vppm $\mathrm{PH}_{3}$ ) and only in the fully dark annealed state. Thus we considered it crucial to investigate the spin state characteristics for light-soaked n-type material as well. In particular, it also seemed important to examine fairly lightly doped samples since then one could greatly perturb the Fermi level position with light-soaking from a position within $0.25 \mathrm{eV}$ of $\mathrm{E}_{\mathrm{C}}$ to nearly midgap (as deep as $\mathrm{E}_{\mathrm{C}}-0.7 \mathrm{eV}$ ). In this manner one might hope to see a "cross-over" between the doped and undoped behaviors. Such results could be quite important for resolving the fundamental nature of metastable defects in a-Si:H. 


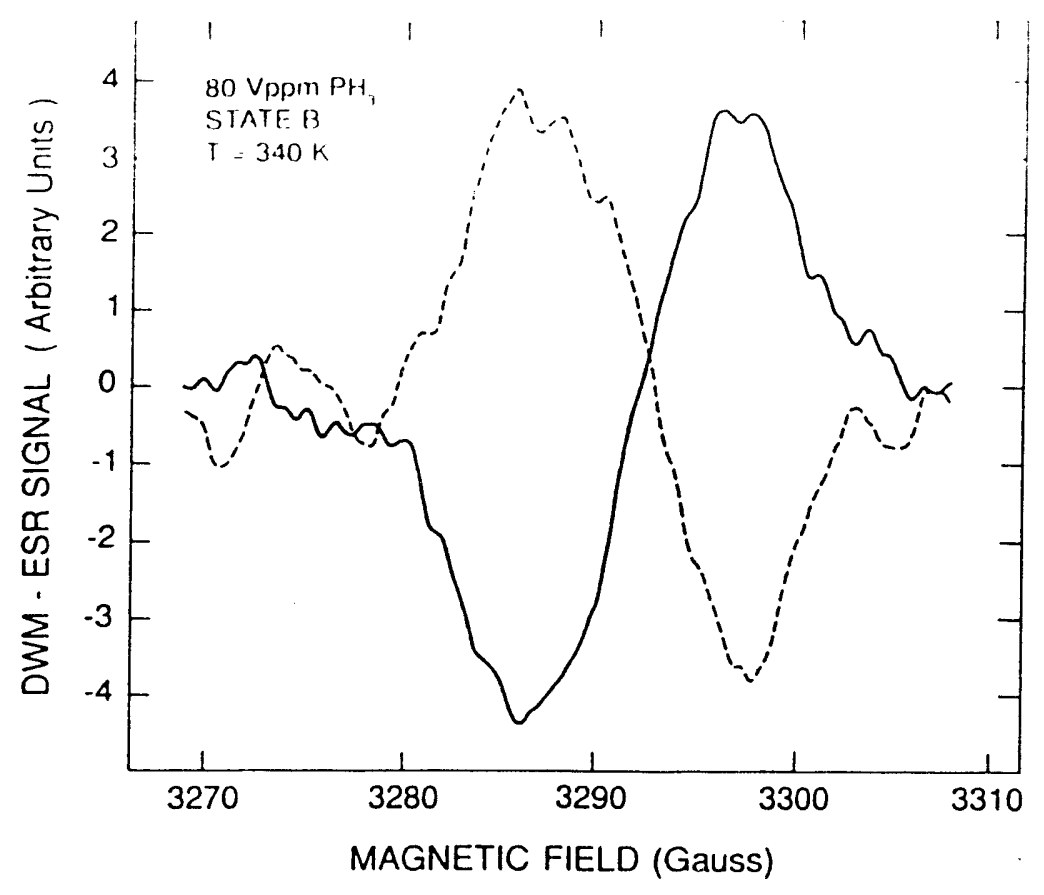

FIG. 12. DWM-ESR spectra of light-soaked (200 hours), n-type a-Si:H (80 Vppm $\mathrm{PH}_{3}$ ) taken at $340 \mathrm{~K}$. The spectra were averaged over 367 magnetic field scans with a modulation field of $12.5 \mathrm{G}$. The two curves shown correspond to the signals in-phase with the $3 \mathrm{~Hz}$ bias voltage modulation (dashed curve) and at $90^{\circ}$ (full curve) with respect to the modulated bias voltage. The bias modulation for these data alternated between a $-1 \mathrm{~V}$ and a $-6 \mathrm{~V}$ level.

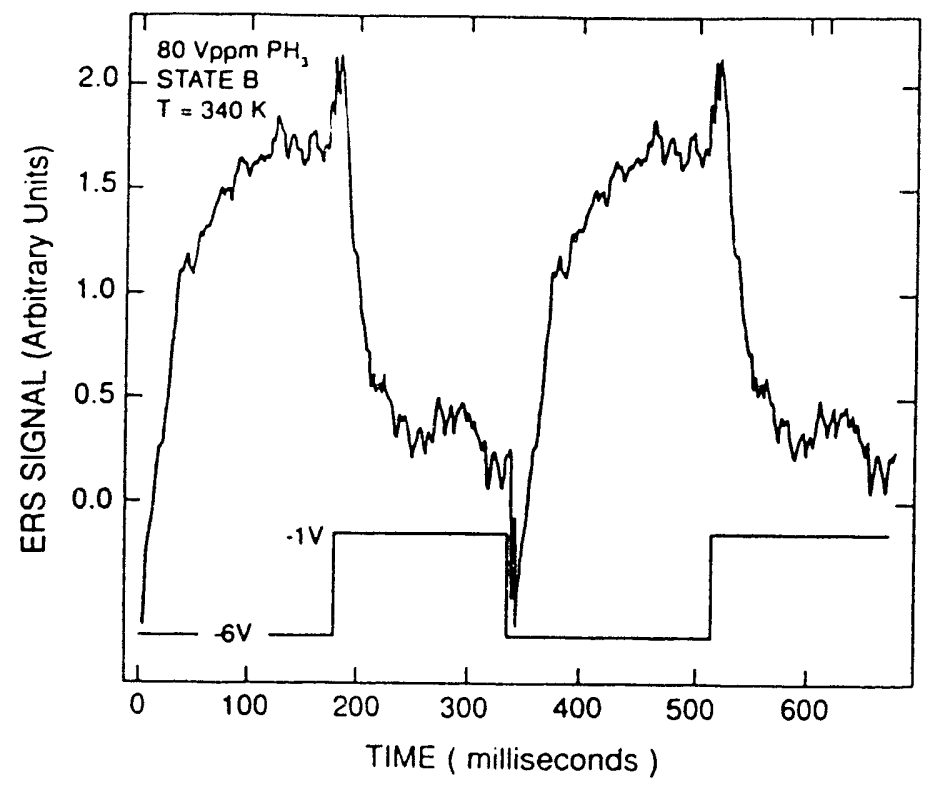

FIG. 13. Spin transient signal of same light soaked state of sample as in Fig. 12, again at $340 \mathrm{~K}$. In this case the magnetic field was kept fixed at $3297 \mathrm{G}$. The data were averaged over $2.25 \times 10^{5}$ sweeps with an $8 \mathrm{~ms}$ time constant. The corresponding modulated bias voltage is depicted at the bottom of the figure. 


\subsection{EXPERIMENTAL RESULTS}

Typical DWM-ESR spectra are shown in Fig. 12 for an $80 \mathrm{Vppm} \mathrm{PH}_{3}$ doped sample in state $B$. This figure exhibits both the in-phase and quadrature signals which appear to have identical $g$ factors and linewidths within the experimental error. The sign of the in-phase signal always appears inverted with respect to a normal ESR absorption signal. This indicates that more spins are present when a larger negative bias is applied to the sample. This implies that the $D^{-} / D^{\circ}$ transition is predominant as trapped gap state electrons are thermally emitted from defects. This agrees with the previous DWM-ESR studies on ntype samples.

The quadrature phase signal is also surprisingly large and inverted with respect to the in-phase signal. This indicates a significant phase lag of the spin signal relative to the charige in applied bias. For this $3 \mathrm{~Hz}$ bias modulation frequency at $340 \mathrm{~K}$, this implies a spin signal phase shift, $\phi_{S}$ of approximately $-50^{\circ}$. This phase shift is observed to decrease as the temperature is raised, consistent with the fact that rates increase with increasing temperature.

Nonetheless, this degree of phase lag seems somewhat surprising. To study this in more detail, we also recorded the spin signal directly as a function of time at constant magnetic field. (We should note that such measurements are extremely difficult to carry out, requiring the signal averaging of several hundred thousand transients over several days.) These data are exhibited in Fig. 13 and are found to agree both in magnitude, sign, and phase shift angle with the spectra given in Fig. 12 (in this case the phase shift angle is calculated to be $-44^{\circ}$ ).

We can compare these results directly with the corresponding capacitance transients observed under identical conditions on the same sample. Figure 14(a) displays the capacitance signal versus time (solid circles) as the applied bias is modulated as indicated in the figure. This capacitance transient can be converted into a deep charge transient by the numerica: simulation of the capacitance data. Such capacitance transient simulations were developed some time ago in order to interpret capacitance DLTS spectra on a-Si:H samples $[14,32]$. The capacitance transient obtained by this simulation is shown as the solid line in Fig. 14(a).

To obtain this simulation we assumed a broad deep defect band, peaked near $\mathrm{E}_{\mathrm{C}}-0.85 \mathrm{eV}$, and adjusted its width and magnitude until we obtained agreement with: (1) drive level derived charge densities at several temperatures, 

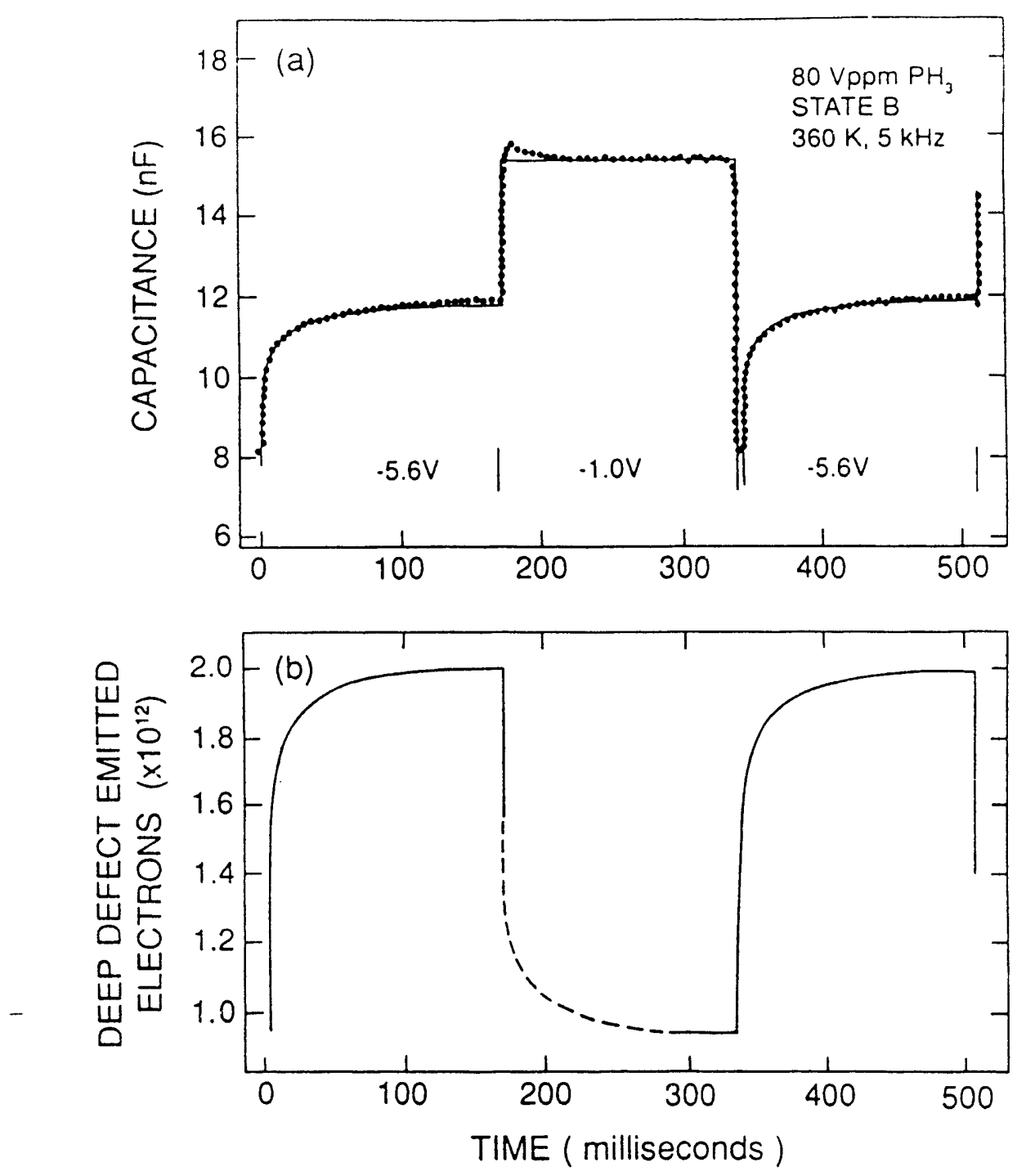

FIG. 14. (a) Measured (full circles) and calculate (solid lines) $5 \mathrm{kHz}$ capactiance transients of a partially light-soaked state (48 hrs.) of the $80 \mathrm{Vppm}^{\mathrm{PHH}} 3$ doped sample. The measurement temperature was $360 \mathrm{~K}$ and the corresponding levels ofthe $3 \mathrm{~Hz}$ modulation voltage are indicated in the figure.

(b) Calculated number of deep defect electrons responding to the modulated bias voltage shown in (a). The full curve corresponds to emission processes and the dashed curve to capture processes. 
and (2) the long time asymptotic values of the capacitance during both the low and high reverse bias phases of the applied voltage. The Fermi level position was also matched to the activation energy of the ac conductivity. We thus obtained quite good agreement to the capacitance transient signal. The resultant deep defect charge variation was then also calculated from this simulation and is displayed in Fig. 14(b). While we don't expect the details of $g(E)$ to be necessarily well reproduced by this method, we have confirmed that the time dependence of the total integrated charge in the deep defects is quite accurate and insensitive to such details of the calculations.

The results of these simulations thus allow us to compare the magnitudes and phase angles of the spin signals with those of the corresponding charge signals. A summary of these comparisons for two samples (a $10 \mathrm{Vppm} \mathrm{PH}_{3}$ doped sample and a $80 \mathrm{Vppm} \mathrm{PH}_{3}$ doped sample) in a variety of metastable states is given in Fig. 15 and Table I. Figure 15 indicates that there exists quite good agreement between the magnitudes of the modulated spin and deep-defect charge signals. However, the phase angles of the calculated charge transients are much smaller than those measured for the spin transients (see Table I).

In addition, the following trends were also noted: (1) The spin phase angle lag decreases with increased $\mathrm{PH}_{3}$ doping; (2) For a given composition, $\phi_{\mathrm{s}}$ decreases with increasing temperature; and (3) The magnitude of the spin signal increases with increasing $\mathrm{PH}_{3}$ doping.

\subsection{DISCUSSION}

We have determined that all of the DWM-ESR results on n-type samples appear qualitatively similar and quite different from those obtained previously on intrinsic samples. [16] That is, all of the observed signals imply the predominance of the $D^{-} / D^{\circ}$ transition and a spin signal that agrees quite well in magnitude with the charge signal. This applies even for the most lightly doped sample in the light-soaked state (where $\mathrm{E}_{\mathrm{F}}$ lies more than $0.6 \mathrm{eV}$ below $\mathrm{E}_{\mathrm{C}}$ ). On the other hand, the existence of the phase lag between the spin and charge signals, which increases as the doping level is decreased, may be the precursor to the qualitatively different behavior observed in the intrinsic samples.

Indeed, the phase lag between the spin and charge signals is quite difficult to explain. At this point we can offer two possible sources for this. One possibility is that, in addition to switching between the $\mathrm{D}^{-}$and $\mathrm{D}^{\circ}$ charge states, 
FIG. 15. Comparison of the magnitudes of the DWM-ESR and the charge modulation signal for two $\mathrm{PH}_{3}$ doped samples and for a variety of metastable states. Here we display the signals in the same absolute units. Solid symbols correspond to state $A$, open symbols to state $B$, the dotted triangle to a partially annealed light-soaked state, and the crossed circle to a partially light-soaked state. Note that signals magnitudes are plotted in absolute numbers rather than number densities; thus, the relative densities in state $B$ are actually much greater than in state $A$, however the depletion width is smaller in state $B$. The solid line indicates a 1-to-1 correspondence between the 2 signal types.

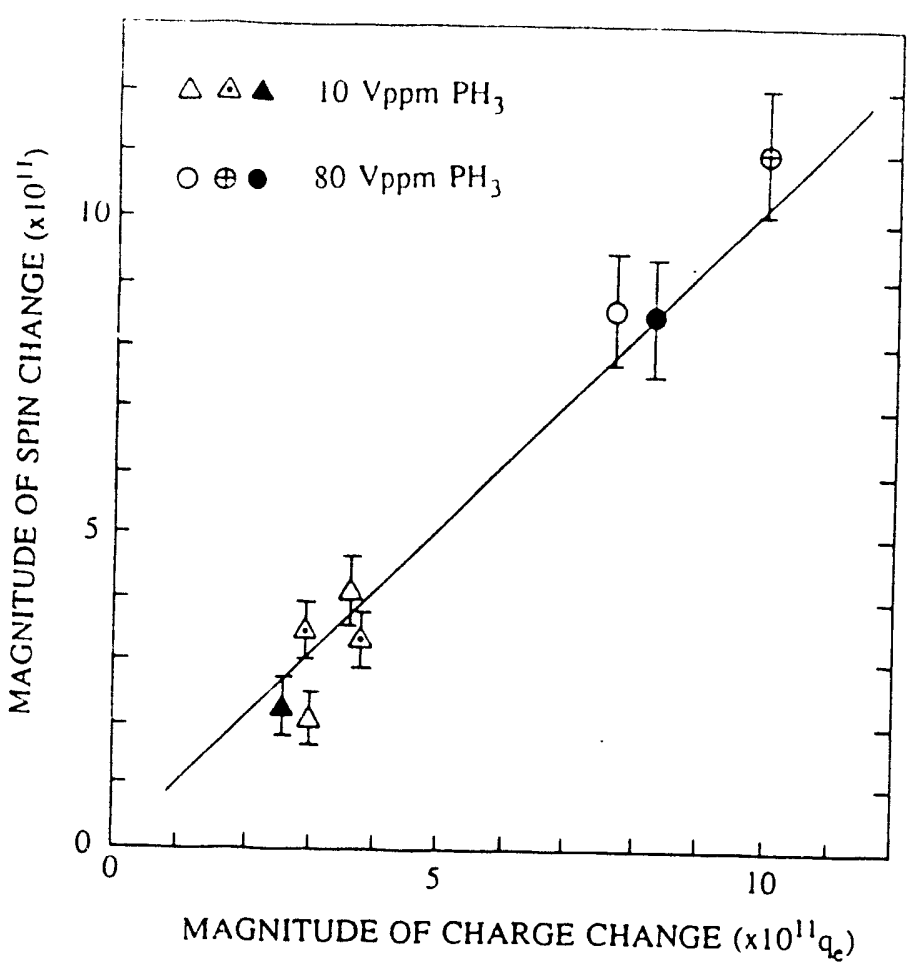

TABLE I. Measured values of the magnitude of modulated spin signal., $\delta \mathrm{N}_{S}$, its phase angle relative to the applied bias, $\phi_{S}$, along with the calculated values of the charge modulation magnitudes, $\delta N_{Q}$, and phase angles, $\phi_{Q}$ obtaineu' from the analysis of the capacitance transient data. The state denoted as $A^{\prime}$ corresponds to a partially annealed light-soaked state, and the state $B^{\prime}$ to a partially light-soaked state.

\begin{tabular}{|c|c|c|c|c|c|c|c|}
\hline Composition & State & $E_{\sigma}(e V)$ & $\begin{array}{c}\text { Temperature } \\
\text { (K) }\end{array}$ & ${ }_{\left(10^{11}\right.}^{\delta N_{s}}$ Sins $)$ & $\begin{array}{c}\phi_{s} \\
\text { (degrees) }\end{array}$ & $\begin{array}{l}\text { Calculated } \\
\delta \mathrm{N}_{\mathrm{Q}}\left(10^{11}\right. \\
\text { electrons })\end{array}$ & $\begin{array}{l}\text { Calculated } \\
{ }^{\phi_{Q} \text { (degrees) }}\end{array}$ \\
\hline \multirow{5}{*}{$\begin{array}{l}10 \mathrm{Vppm} \\
\mathrm{PH}_{3} / \mathrm{SiH}_{4}\end{array}$} & \multirow[t]{2}{*}{ B } & \multirow[t]{2}{*}{0.61} & 340 & $2.1 \pm 0.3$ & -68 & 3.0 & -8.9 \\
\hline & & & 360 & $4.1 \pm 0.6$ & -41 & 3.7 & .6 .8 \\
\hline & \multirow[t]{2}{*}{$A^{\prime}$} & \multirow[t]{2}{*}{0.35} & 340 & $3.4 \pm 0.4$ & -59 & 3.0 & .7 .0 \\
\hline & & & 360 & $3.3 \pm 0.4$ & -36 & 3.8 & .60 \\
\hline & $A$ & 0.28 & 340 & $2.2 \pm 0.4$ & -68 & 2.5 & .10 .0 \\
\hline \multirow{3}{*}{$\begin{array}{l}80 \mathrm{Vppr} \\
\mathrm{PH}_{3} / \mathrm{S}_{1} \mathrm{H}_{4}\end{array}$} & $\mathrm{~B}$ & 0.56 & 340 & $8.5 \pm 0.7$ & .50 & 7.7 & .8 .7 \\
\hline & $\mathrm{B}^{\prime}$ & 0.33 & 360 & $11 \pm 0.9$ & $.4 !$ & 9.8 & .8 .3 \\
\hline & A & 0.28 & 340 & $8.4 \pm 07$ & -45 & 8.2 & .57 \\
\hline
\end{tabular}


we also have some population of $\mathrm{D}^{+}$states following the emission phase. In that case thie subsequent capture of electrons would both create unpaired spins as charge is captured into the $\mathrm{D}^{+}$and subsequently destroy spin as charge is captured into the $\mathrm{D}^{0}$ states. This could indeed lead to a significant phase lag for the spin signal compared to the total charge change. We have verified that we can roughly reproduce the experimentally observed phase angle values for both spin and charge in detailed modeling of such situations; however, we also find that this will lead to a considerable suppression of the magnitude of the spin signal compared to the charge signal (by about a factor of two). This explanation thus appears to be inconsistent with the stated accuracy in our calibration of the magnitudes of spin and charge signals.

A second possibility is that the spin signal at $g=2.0055$ that we observe does not occur immediately after capture. Rather, there exists an intermediate state with a somewhat different $g$-factor and/or broader lineshape. Since these spin signals are so weak, it is quite crucial that we optimize the parameters on our ESR spectrometer to the expected position and lineshape. Any significant change in these characteristics would render the spin signal invisible. Thus we would observe a spin signal that appears only after it has had time to relax into its equilibrium configuration. This would produce a large phase shift in the spin signal but would not appreciably affect its overall magnitude. The charge signal, on the other hand, would be present independent of such details so that it would exhibit a much smaller phase shift.

\subsection{SUMMARY OF KEY FINDINGS AND RECOMMENDATIONS FOR FUTURE STUDY}

a. Impurity Modulation Studies

Our studies of samples with modulated impurity profiles coupled with drive-level capacitance profiling measurements have extended the sensitivity and reliability of trying to establish correlations between light-induced degradation and extrinsic compositional factors. In this manner we have established a clear correlation between carbon contamination at levels as low as 0.1 at.\% and a significant degree of increased light-induced defect creation. Our findings have recently been cited as possible evidence for a newly proposed mechanism for light-induced defect creation. That mechanism extrapolates 
calculations for the DX center in III-V semiconductors to an impurity based model of deep defect creation in a-Si:H. [31]

Clearly there are a number of obvious extensions for this work. Other impurities that are common contaminants of a-Si: $\mathrm{H}$ should be investigated in a similar manner, including at the very least oxygen and nitrogen. Also, it would be very important to extend the range of carbon impurity concentration to the regime at or below 0.01 at.\%. This would requre samples with much lower levels of unintentional impurities.

We were also able to resolve the widths of the transition regions between low and high defect regions to a fairly high accuracy (to $\pm 50 \AA$ in one case). This aspect of our experimental capabilities may prove to be quite useful in some planned experiments to correlate the diffusion of hydrogen with the annealing behavior of such light-induced defects.

b. Defect Reactions in Doped Samples

In our studies of metastable states in phosphorus doped a-Si:H samples, we discussed how a measurement of the changes in the density of occupied bandtail states compared to changes in the density of metastable defects could be used to distinguish different classes of microscopic defect reactions that have been proposed for doped a-Si:H. In this manner, we found that dopants could be de-activated by light-soaking somewhat independently of the creation of deep defects, at least in more lightly doped samples. For all samples studied we also found that quench cooling increased the effective doping efficiency without any measurable change in deep defects. We thus proposed some possible microscopic processes that might account for these results. Several of the examples we discussed involved bond switching and motion of hydrogen.

We also found a linear correlation between the concentration of deep defects and the phosphorus doping level in the regime below $100 \mathrm{Vppm}$, not the $[P]^{1 / 2}$ dependence that had been reported previously. [28] We believe this again reflects the quasi-independence of dopant activation from deep defect creation. Our conclusions agree substantially with those reached by recent studies of lithium interstitial doping in a-Si:H. [30]

The types of studies carried out in this report could readily be extended to boron-doped or even intrinsic a-Si:H samples. In the latter case one could monitor Fermi level shifts together with the density of states at the Fermi level, $g\left(E_{F}\right)$, instead of the occupied bandtail states. One could then make a 
quantitative comparison between the changes in the occupation of states near $E_{F}$ with the changes in metastable deep defects. Such a detailed comparison of such quantities has never been attempted and might prove quite informative toward assessing the validity of the classes of microscopic defect reactions that have been proposed.

\section{c. The Spin/Charge Relation for Metastable Defects in Doped Samples}

Using depletion width modulated ESR spectroscopy together with junction capacitance techniques, we investigated the thermally excited electronic transitions for deep defect states for lightly doped a-Si:H samples in a variety of metastable states. These techniques allowed us to enumerate the change in total spin and charge associated with these transitions. In all cases we found that the magnitude of the modulated spin to charge ratio was very nearly unity. This result was consistent with earlier studies of n-type doped a-Si:H [13] and indicates that the $\mathrm{D}^{-} / \mathrm{D}^{\circ}$ transition is dominant in all cases.

However, we also observed a previously unreported "quadrature" DWMESR signal indicating a substantial phase lag between the spin signal and the modulation of the junction applied bias. To verify this we obtained, for the first time, a recording of the actual spin transient $v$ s. time for one sample. A detailed examination of the capacitance transients recorded under identical conditions indicated a much smaller phase lag for the charge signal involved in the trapping and de-trapping of charge from deep states. The reasons for this relative phase difference between the spin and charge signals is not yet understood. We suggest that it may indicate an intermediate state that is involved in the thermal emission and/or capture of electrons into the D-center in a-Si:H.

This kind of experiment addresses very fundamental issues in our understanding of the nature of the deep defect in a-Si:H. Many more studies of this kind are required to fully resolve the questions that have been raised. These should include the study of even more lightly doped samples, the investigation of intrinsic samples in more detail to include the measurement of the actual spin transients, the study of spin signals in more heavily doped samples to study the trapping and de-trapping of electrons into bandtail states, etc. While one would learn a great deal by examining the spin signatures in these cases, the DWM-ESR method suffers from very weak signal levels which require time consuming signal averaging. Nonetheless, we hope to pursue at least a few of the lines of inquiry we have suggested in the near future. 


\subsection{PERSONNEL}

J. David Cohen

John M. Essick

Randall Rasmussen

Thomas Unold

Thomas M. Leen
Principal Investigator/Program Manager

Research Associate: December 1988 to July 1990

Research Associate: September 1989 to April 1991

Research Assistant: April 1988 to April 1991

Research. Assistant: January 1989 to December 1990

\subsection{PUBLISHED PAPERS BASED ON SERI SUBCONTRACT WORK}

1) Thomas Unold and J. David Cohen, "The carbon impurity dependence of light induced metastable effects in hydrogenated amorphous silicon", J. NonCryst. Solids $\underline{114}, 603$ (1989).

2) T.M. Leen, J.D. Cohen, and A.V. Gelatos, "Capacitance Studies of Metastable States in Light-Soaked, Quench-Cooled, and Bias-Annealed n-type Hydrogenated Amorphous Silicon", Mat. Res. Soc. Symp. Proc. 192, 707 (1990).

3) Thomas Unold and J. David Cohen, "Trace Impurities and Metastable States in a-Si:H", Mat. Res. Soc. Symp. Proc. 192, 719 (1990).

4) - Thomas Unold and J. David Cohen, "Enhancement of light-induced degradation in hydrogenated amorphous silicon due to carbon impurities", Appl. Phys. Lett. 58,723 (1991).

5) J.D. Cohen and T. Unold, "The effects of impurities on the light-induced degradation of hydrogenated amorphous silicon", Solar Cells 30293 (1991).

6) J. David Cohen and Thomas M. Leen, "Investigation of defe $i$ reactions involved in metastability of hydrogenated amorphous silicon", AIP Conference Proceedings, in press.

7) R.J. Rasmussen, J.D. Cohen, and J.M. Essick, "An investigation of spin and charge states associated with metastable defects in n-type hydrogenated amorphous silicon", Mat. Res. Soc. Symp. Proc., in press. 


\subsection{REFERENCES}

1. R. Crandall, D. Carlson, A. Catalano, and H.A. Weakliem, Appl. Phys. Lett. 44, 200 (1984).

2. D.E. Carlson, A. Catalano, R.V. D'Aiello, C.R. Dickson, and R.S. Oswald, in Optical Effects in Amorphous Semiconductors, ed. by P. C. Taylor and S. G. Bishop (AIP Conf. Proc., No. 120, NewYork, 1984), p.

3. A. Skumanich, N.M. Amer, and W.B. Jackson, Phys. Rev. B31, 2263 (1985).

4. A. Skumanich and N.M. Amer, Phys. Rev. B37 8465 (1988).

5. C.E. Michelson, A.V. Gelatos, and J.D. Cohen, Appl. Phys. Lett. 48, 412 (1985).

6. K.K. Mahavadi, K. Zellama, J.D. Cohen, and J.P. Harbison, Phys. Rev. B35, 7776 (1987).

7. A.V. Gelatos, K.K. Mahavadi, J.D. Cohen, and J.P. Ficibison, Appl. Phys. Lett. 53,403 (1988).

8. J. David Cohen and Avgerinos V. Gelatos, in Amorphous Silicon and Related Materials, ed. by H. Fritzsche (World Scientific, Singapore, 1988), p. 475.

9. H. Dersch, J. Stuke, and Beichler, Phys. Status Solidi (b) 105, 265 (1981).

10. For a recent review see M. Stutzmann and D.K. Biegelsen, Ibid, p. 557.

11. R.A. Street and D.K. Biegelsen, Solid State Commun. 33, 1159 (1980).

12. N.M. Johnson and D.K. Biegelsen, Phys. Rev. B31, 4066 (1985).

13. J.D. Cohen, J.P. Harbison, K.W. Wecht, Phys. Rev. Lett. 48, 109 (1982).

14. D.V. Lang, J.D. Cohen, and J.P. Harbison, Phys. Rev. B25, 5285 (1982).

15. W.B. Jackson and N.M. Amer, Phys. Rev. B25, 5559 (1982).

16. J.M. Essick and J.D. Cohen, Phys. Rev. Lett. 64,3062 (1990).

17. M. Stutzmann, W.B. Jackson, and C.C. Tsai, Phys. Rev. B32 23 (1985).

18. A.V. Gelatos, K.K. Mahavadi, J.D. Cohen, and J.P. Harbison, Appl. Phys. Lett. $\underline{53}, 403$ (1988).

19. J. Singh and A. Madhukar, Solid State Comm. 41, 241 (1982). 
20. M.B. Schubert, H.D. Mohring, E. Lotter, and G.H. Bauer, IEEE Transactions on Electron Devices 36, 2863 (1989).

21. R.A. Street, D.K. Biegelsen, W.B. Jackson, N.M. Johnson, and M. Stutzmann, Phil. Mag. B52, 235 (1985).

22. M. Stutzmann, Phys. Rev. B35, 9735 (1987).

23. A.V. Gelatos and J.D. Cohen, Mat. Res. Soc. Symp. Proc. 118141 (1988).

24. Z E. Smith, S. Aljishi, D. Slobodin, V. Chu, S. Wagner, P.M. Lenahan, R.R. Arya, and M.S. Bennett, Phys. Rev. Lett. 57 2450 (1986).

25. T.M. Leen, J.D. Cohen, and A.V. Gelatos, Mat. Res. Soc. Symp. Proc. 192 707 (1990).

26. J. Zhu, N.M. Johnson, and C. Herring, Phys. Rev. B411, 12354 (1990).

27. D.V. Lang, J.D. Cohen, and J.P. Harbison, Phys. Rev. Lett. $\underline{48} 421$ (1982).

28. R.A. Street, D.K. Biegelsen, W.B. Jackson, N.M. Johnson, and M. Stutzmann, Phil. Mag. B52, 235 (1985).

29. See, for example, M. Stutzmann, Phil. Mag. Lett. 53 L15 (1986).

30. K. Pierz, W.Fuhs, H. Mell, Philos. Mag. B63, 123 (1991).

31. - D. Redfield and R.H. Bube, Phys. Rev. Lett. 65, 464 (1990).

32. J.D.Cohen and D.V. Lang, Phys. Rev. B25, 5321 (1982). 


\begin{tabular}{|c|c|c|c|}
\hline $\begin{array}{l}\text { Document Control } \\
\text { Page }\end{array}$ & $\begin{array}{l}\text { 1. SERI Report No. } \\
\text { NREL/TP-214-4592 }\end{array}$ & $\begin{array}{l}\text { 2. NTIS Accession No. } \\
\text { DE92001178 }\end{array}$ & 3. Recipient's Accession No. \\
\hline \multirow{2}{*}{\multicolumn{3}{|c|}{$\begin{array}{l}\text { 4. Title and Subtitle } \\
\text { Investigations of the Origins of Metastable Light-Induced Changes in } \\
\text { Hydrogenated Amorphous Sil: on }\end{array}$}} & $\begin{array}{l}\text { 5. Publication Date } \\
\text { December } 1991\end{array}$ \\
\hline & & & 6. \\
\hline $\begin{array}{l}\text { 7. Author(s) } \\
\text { J.D. Cohen }\end{array}$ & & & 8. Performing Organization Rept. No. \\
\hline \multirow{2}{*}{\multicolumn{3}{|c|}{$\begin{array}{l}\text { 9. Performing Organization Name and Address } \\
\text { Department of Physics and Materials Science Institure } \\
\text { University of Oregon } \\
\text { Eugene, Oregon }\end{array}$}} & $\begin{array}{l}\text { 10. Project/TaskWork Unit No. } \\
\text { PV141101 }\end{array}$ \\
\hline & & & $\begin{array}{l}\text { 11. Contract (C) or Grant (G) No. } \\
\text { (C) XM-8-18061-1 } \\
\text { (G) }\end{array}$ \\
\hline \multirow{2}{*}{\multicolumn{3}{|c|}{$\begin{array}{l}\text { 12. Sponsoring Organization Namie and Address } \\
\text { National Renewable Energy Laboratory } \\
1617 \text { Cole Blvd. } \\
\text { Golden, CO } 80401-3393\end{array}$}} & $\begin{array}{l}\text { 13. Type of Report \& Period Covered } \\
\text { Technical report, } \\
1 \text { April } 1988-31 \text { March } 1991 \\
\end{array}$ \\
\hline & & & 14. \\
\hline \multicolumn{4}{|c|}{$\begin{array}{l}\text { 15. Supple mentary Notes } \\
\text { NREL technical monitor: B. Stafford, (303) } 231-7126\end{array}$} \\
\hline \multicolumn{4}{|c|}{ 16. Abstract (Limit: 200 words) } \\
\hline \multicolumn{4}{|c|}{ 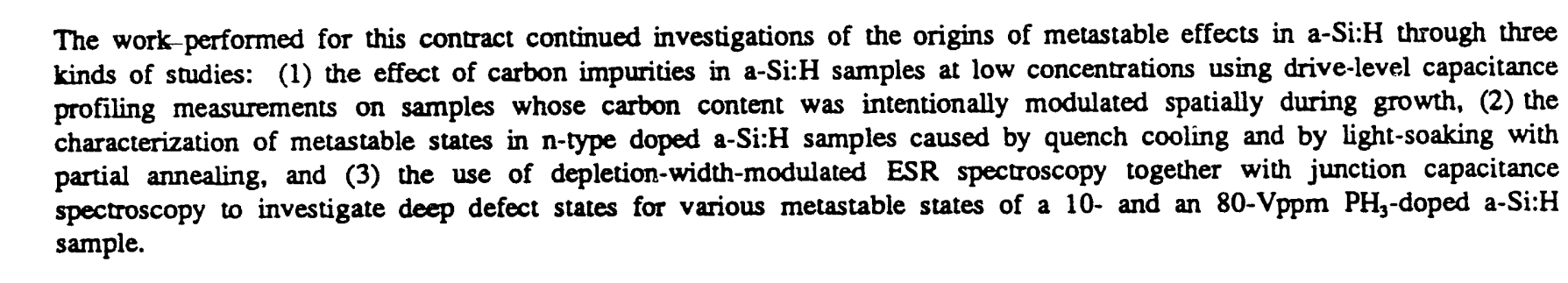 } \\
\hline \multicolumn{4}{|c|}{$\begin{array}{l}\text { 17. Document Analysis } \\
\text { a. Descriptors } \\
\text { photovoltaics ; solar cells ; amorphous silicon } \\
\text { b. Identifiers/Open-Ended Terms }\end{array}$} \\
\hline \multicolumn{4}{|l|}{$\begin{array}{l}\text { c. UC Categories } \\
271\end{array}$} \\
\hline \multirow{2}{*}{\multicolumn{2}{|c|}{$\begin{array}{l}\text { 18. Availability Statement } \\
\text { National Technical Information Service } \\
\text { U.S. Department of Commerce } \\
5285 \text { Port Royal Road } \\
\text { Springfield, VA } 22161\end{array}$}} & & $\begin{array}{l}\text { 19. No. of Pages } \\
43\end{array}$ \\
\hline & & & $\begin{array}{l}\text { 20. Price } \\
\text { A03 }\end{array}$ \\
\hline
\end{tabular}

Form No. 0069E (6-30-87) 

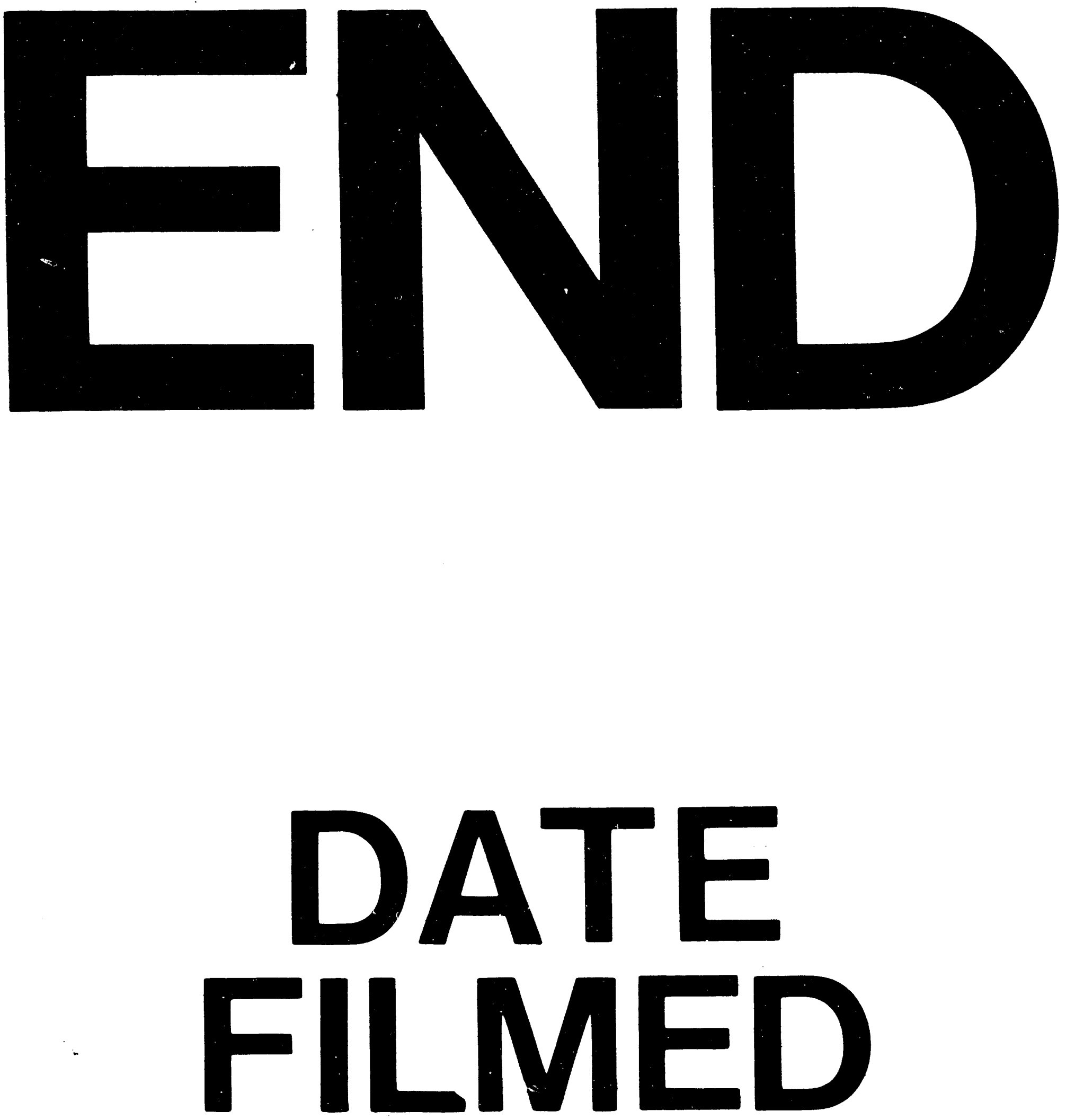

1

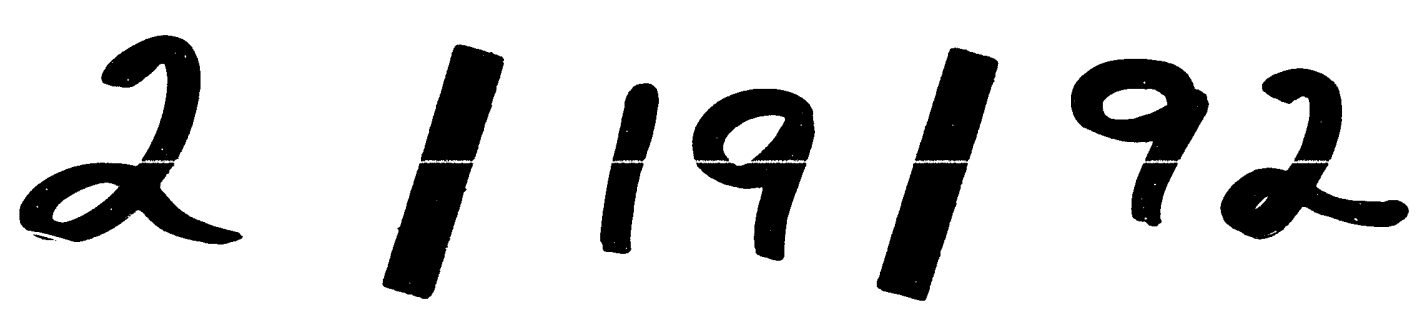


